\title{
GUÍAS DE PRÁCTICA CLÍNICA DE LA SERV: MANEJO DE LAS COMPLICACIONES OCULARES DE LA DIABETES. RETINOPATÍA DIABÉTICA Y EDEMA MACULAR
}

\author{
GUIDELINES OF CLINICAL PRACTICE OF THE SERV: \\ MANAGEMENT OF OCULAR COMPLICATIONS OF DIABETES. \\ DIABETIC RETINOPATHY AND MACULAR OEDEMA
}

\author{
PAREJA-RÍOS A ${ }^{1}$, SERRANO-GARCÍA MA ${ }^{1}$, MARRERO-SAAVEDRA MD ${ }^{2}$, \\ ABRALDES-LÓPEZ VM ${ }^{3}$, REYES-RODRÍGUEZ MA MA $^{4}$ CABRERA-LÓPEZ F ${ }^{5}$, LÓPEZ-GÁLVEZ M ${ }^{6}$, \\ CARDONA-GUERRA $P^{5}$, ABREU-REYES $\mathrm{P}^{7}$, QUIJADA-FUMERO E ${ }^{1}$, CORONADO-TOURAL A ${ }^{8}$, \\ GUTIÉRREZ-SÁNCHEZ E ${ }^{9}$, GIL-HERNÁNDEZ MA ${ }^{7}$, VALLS-QUINTANA P ${ }^{4}$, MARÍN-OLMOS F ${ }^{10}$, \\ NAVARRO-ALEMANY R ${ }^{11}$
}

\section{RESUMEN}

Objetivo: La diabetes mellitus está considerada como la causa más frecuente de ceguera en la población activa en los países industrializados, siendo el edema macular diabético la causa más frecuente de disminución de la agudeza visual y la retinopatía diabética proliferante la responsable de los déficit visuales más severos. Por ello hemos intentado establecer una guía de actuación clínica cuyo propósito es proporcionar unas directrices que sirvan de orientación para el tratamiento de la reti-

\begin{abstract}
Objective: Diabetes mellitus is considered the most common cause of blindness in the working population of industrialized countries, with diabetic macular edema being the most common cause of decreased visual acuity and proliferative diabetic retinopathy (PDR) being responsible for the most severe visual deficits. We have therefore tried to establish a guide for clinical intervention whose purpose is to provide orientation on the treatment of diabetic retinopathy and its complications. This is necessary at
\end{abstract}

Recibido: 20/7/09. Aceptado: 18/9/09.

Sociedad Española de Retina y Vítreo.

1 Doctor en Medicina. Hospital Universitario de Canarias, La Laguna. Tenerife. España.

2 Doctor en Medicina. Hospital Universitario de Gran Canaria Dr. Negrín, Las Palmas de Gran Canaria. España.

3 Doctor en Medicina. Complejo Hospitalario Universitario de Santiago de Compostela. Instituto Tecnológico de Oftalmología. Santiago de Compostela. España.

${ }^{4}$ Licenciado en Medicina. Hospital Universitario de Gran Canaria Dr. Negrín, Las Palmas de Gran Canaria. España.

5 Doctor en Medicina. Complejo Hospitalario Materno-Insular. Las Palmas de Gran Canaria. España.

6 Doctor en Medicina. Hospital Clínico Universitario de Valladolid. Instituto de Oftalmobiología Aplicada. Valladolid. España.

7 Doctor en Medicina. Hospital Universiario Sra. de Candelaria. Tenerife. España.

8 Doctor en Medicina. Clínica de Cirugía Ocular (CCO). Madrid. España.

9 Licenciado en Medicina. Clínica Piñero. Sevilla. España.

10 Doctor en Medicina. Hospital Clínico Universitario de Valencia. Valencia. España.

11 Licenciado en Medicina. Instituto de Microcirugía ocular (IMO). Barcelona. España.

Los autores no tienen interés comercial ni han recibido apoyo económico.

Correspondencia:

Alicia Pareja Ríos

Hospital Universitario de Canarias

C/. Ofra, s/n

38320 La Laguna (Tenerife)

España

E-mail: aparejar@gmail.com 
nopatía diabética y sus complicaciones. Esto se hace necesario en un momento en el que han aparecido numerosas alternativas terapéuticas cuyo papel aún no está completamente definido.

Método: Un grupo de expertos retinólogos seleccionados por la SERV han evaluado los resultados publicados sobre las distintas opciones terapéuticas que existen en la actualidad, en base a lo cual se sugieren líneas de actuación según el grado de retinopatía diabética que presenta el paciente y la presencia o no de edema macular.

Resultados: El tratamiento princeps de la RDP es la panretinofotocoagulación (PFC). El tratamiento de elección en el edema macular diabético clínicamente significativo sin signos de tracción vítreo macular continúa siendo la fotocoagulación focal/rejilla. La cirugía retinovítrea tiene así mismo sus indicaciones en ambas afecciones. Se discute el uso de fármacos antiangiogénicos.

Conclusión: La laserterapia es efectiva en el manejo de la RD y del EMD. El papel de los antiangiogénicos aún no está suficientemente definido.

Palabras clave: Retinopatía diabética, edema macular diabético, tomografía de coherencia óptica, fotocoagulación, vitrectomía, fármacos antiangiogénicos intravítreos. a time when many treatment options have emerged whose role is not yet fully defined.

Method: A group of expert retina specialists selected by the SERV (Vitreous-Retina Spanish Society) assessed the published results of different treatment options currently available, suggesting lines of action according to the degree of diabetic retinopathy present and the presence or absence of macular edema.

Results: PDR is primarily treated with pan-retinal photocoagulation. For clinically significant diabetic macular edema without signs of vitreomacular traction, the treatment of choice continues to be focal/grid photocoagulation. Similarly, retinovitreal surgery is indicated for both conditions. The use of antiangiogenic drugs was also analyzed but remains inconclusive.

Conclusion: Laser therapy is effective in the management of diabetic retinopathy and diabetic macular edema. The role of antiangiogenics is not yet sufficiently defined (Arch Soc Esp Oftalmol 2009; 84: 429-450).

Key words: Diabetic retinopathy, diabetic macular oedema, optic coherence tomography, photocoagulation, vitrectomy, intravitreal antiangiogenic drugs.

\section{OBJETIVOS}

El propósito de esta guía de manejo de las complicaciones oculares de la diabetes es presentar unas directrices generales que sirvan de orientación para el tratamiento de la retinopatía diabética y/o el edema macular en un momento en el que están apareciendo nuevas alternativas terapéuticas cuyo papel aún no está bien definido.

Para establecer las directrices se ha realizado una amplia revisión de la literatura y de los protocolos existentes por parte de una comisión nombrada a tal fin por la Sociedad Española de Retina y Vítreo, y se han discutido las distintas opciones terapéuticas disponibles en la actualidad y su indicación más aceptada según el grado de retinopatía diabética que presente el paciente. De esta manera se plantea qué hacer con una retinopatía diabética no proliferante (RDNP) leve, moderada (ambas de control por el oftalmólogo de zona), severa (susceptible de fotocoagulación en algunas ocasiones) o proliferante (control hospitalario). Asimismo se sugiere cómo actuar ante el edema macular diabético. Se discute también el papel de las distintas exploraciones complementarias.

$\mathrm{Su}$ adaptación a los distintos sistemas sanitarios puede suponer la variación de algunos de los aspectos de la misma. Por ejemplo, en las comunidades en las que los Centros de Atención Especializada (CAEs) estén dotados de aparatos de láser, el tratamiento de la retinopatía diabética y del edema macular podrá realizarse en ese ámbito. Derivándose a los hospitales únicamente los casos que sean quirúrgicos. En las comunidades en las que los CAEs carezcan de dicho aparataje todo el tratamiento deberá ser necesariamente hospitalario.

\section{INTRODUCCIÓN}

La Diabetes Mellitus está considerada como la causa más frecuente de ceguera en la población 
activa en los países industrializados, siendo el Edema Macular Diabético la causa más frecuente de disminución de la agudeza visual en los diabéticos y la Retinopatía Diabética Proliferante la responsable de los déficit visuales más severos (1).

El láser ha sido y continúa siendo el tratamiento princeps para las complicaciones oculares de la diabetes. La Panretinofotocoagulación (PFC) consigue evitar la progresión hacia la ceguera en un porcentaje significativo de pacientes. Sin embargo, los resultados del láser son mucho más decepcionantes en el control del edema macular donde sólo se detiene la progresión en un $50 \%$ de los pacientes.

La búsqueda de nuevas alternativas se ha convertido en un objetivo prioritario y aunque los mecanismos patogénicos involucrados en el desarrollo de este proceso aún no son bien conocidos, la implicación del factor de crecimiento vascular endotelial en el mismo ha abierto una nueva vía de investigación. Son numerosas las publicaciones que hablan de la utilidad de la triamcinolona intravítrea y de los antiangiogénicos (antiVEGF) en el control del edema macular diabético y actualmente se han puesto en marcha varios ensayos clínicos en Europa y en EEUU que tratan de evaluar su seguridad y eficacia. Los fármacos antiVEGF se administran por vía intravítrea de forma repetida, lo que en una enfermedad crónica como la diabetes supone una dificultad importante, de ahí que, de forma paralela, se esté evaluando la posibilidad de utilizarlos de forma combinada con el tratamiento láser para que su efecto sea más duradero.

Ante esta situación de cambio e incertidumbre se hace necesario establecer unos criterios uniformes que sirvan de guía en el abordaje de estas complicaciones a la espera de que concluyan estos estudios y podamos disponer de nuevas pautas que permitan mejorar el pronóstico funcional visual de estos pacientes.

\section{CLASIFICACIÓN DE LA RETINOPATÍA DIABÉTICA (RD) Y DEL EDEMA MACULAR (EMD)}

De acuerdo con los resultados de los grandes estudios multicéntricos, la prevención de la ceguera por RD pasa por la realización de revisiones periódicas del fondo de ojo de los pacientes diabéticos de manera que puedan ser tratadas a tiempo las formas con gran riesgo de pérdida severa de visión o incluso de ceguera. Esto exige, además, el empleo de criterios homogéneos de clasificación y tratamiento de la retinopatía diabética. La clasificación propuesta por el Early Treatment Diabetic Retinopathy Study (ETDRS) (2) perseguía este fin y de hecho está considerada como referente a seguir en los ensayos clínicos. Sin embargo, no se utiliza habitualmente en la práctica clínica debido a su complejidad, dado que existen demasiados niveles o estadios, y a que tienen que guardar una correlación con las fotografías estereoscópicas de los 7 campos básicos.

En un intento de conseguir un medio de comunicación para todos, un grupo de expertos (Global Diabetic Retinopathy Project Group-GDRPG-) (3) propuso en el año 2002 una nueva clasificación para la retinopatía diabética de más fácil manejo, que es la Escala Internacional de Severidad de la Retinopatía, y es la que este grupo considera que debería de utilizarse en la práctica clínica. Esta nueva clasificación está basada en los resultados del ETDRS y, por lo tanto, se apoya en la evidencia científica, no pretendiendo desplazar a la clasificación original sino proporcionar una base de manejo sencilla y adecuada a la práctica clínica.

Tabla I. Clasificación de la RD por GDRPG

Sin RD aparente

RD no Proliferativa (RDNP) Leve

RDNP Moderada

RDNP Severa

RDNP Muy Severa:

RDP
Ausencia de microaneurismas $(\mu \mathrm{A})$

Sólo $\mu$ A (fig. 8)

$\mu \mathrm{A}$ asociado a menos de 20 hemorragias $(\mathrm{H})$ intrarretinianas en cada uno de los 4 cuadrantes (C), exudados duros (ED), «exudados» algodonosos (EA), arrosariamiento venoso (AV) en 1 sólo C (fig. 9)

$\mu \mathrm{A}$ junto a uno de los siguientes hallazgos

- Hemorragias intraretinianas severas $(>20)$ en cada uno de los $4 \mathrm{C}$

- Arrosariamiento venoso en $\geq 2 \mathrm{C}$

- Anomalías microvasculares intrarretinianas (AMIR) en $\geq 1 \mathrm{C}$ (fig. 11)

$\mu \mathrm{A}$ junto al menos dos de los hallazgos anteriores

Neovasos (NV) y/o Hemorragia prerretiniana o Hemovítreo (fig. 12). 


\subsection{Clasificación Clínica Internacional de la RD (GDRPG) (tabla I)}

Sin embargo, para el abordaje del edema de mácula la clasificación propuesta por el ETDRS parece ser la más adecuada.

\subsection{Clasificación del Edema Macular (ETDRS) (1985)}

- Sin edema macular.

- Con edema macular (EM): engrosamiento retiniano dentro de 1 diámetro de disco (DD) del centro de la mácula, determinado en el examen estereoscópico con la lámpara de hendidura o con fotografías estereoscópicas a color $\left(30^{\circ}\right)$ (no es EM la

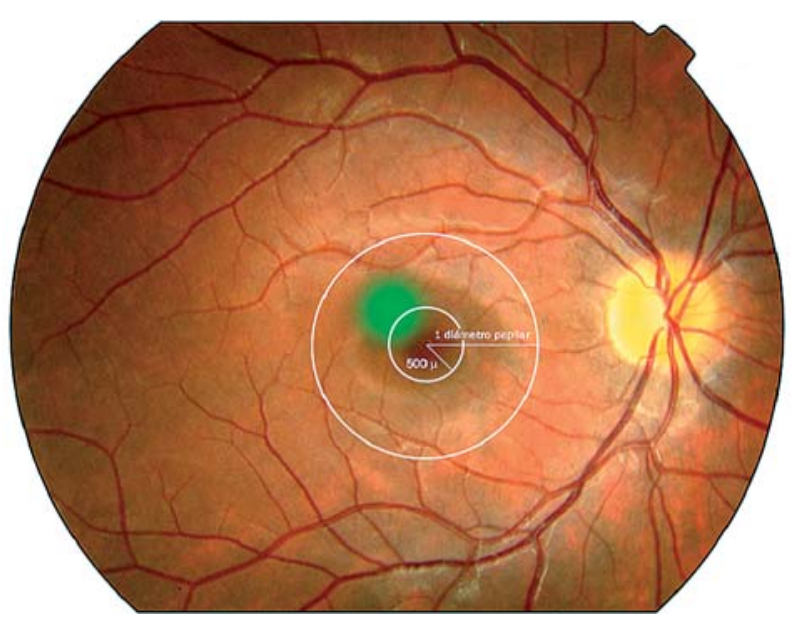

Fig. 1: Engrosamiento retiniano dentro de 500um del centro.

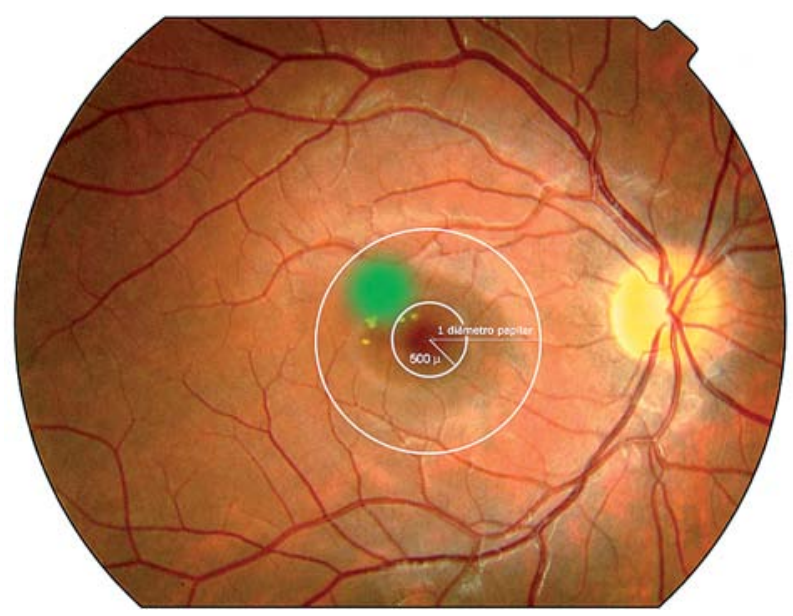

Fig. 2: Exudados duros dentro de $500 \mu \mathrm{m}$ del centro asociado a un engrosamiento de retina adyacente.

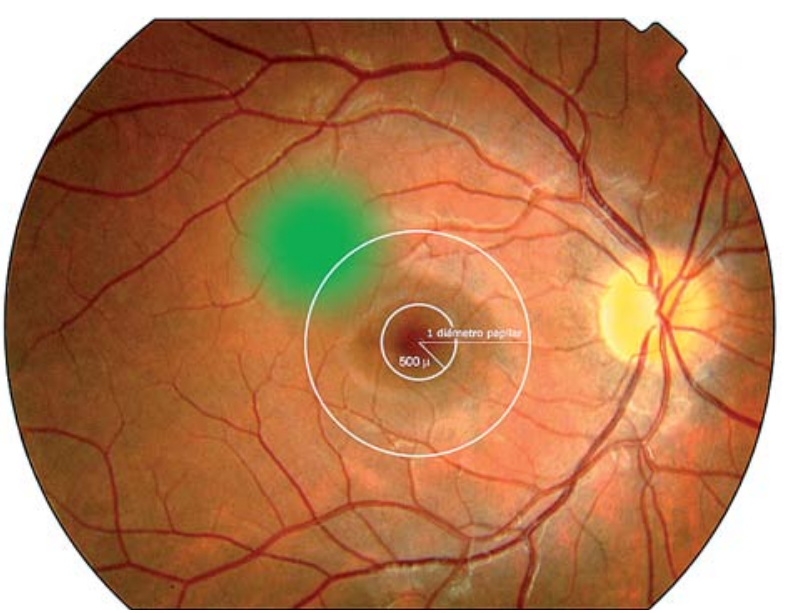

Fig. 3: Engrosamiento retiniano de al menos 1 DD, parte de la cual está a menos de $1 \mathrm{DD}$ del centro.

difusión de fluoresceína en la AFG si no existe engrosamiento). En el ETDRS también se clasificó
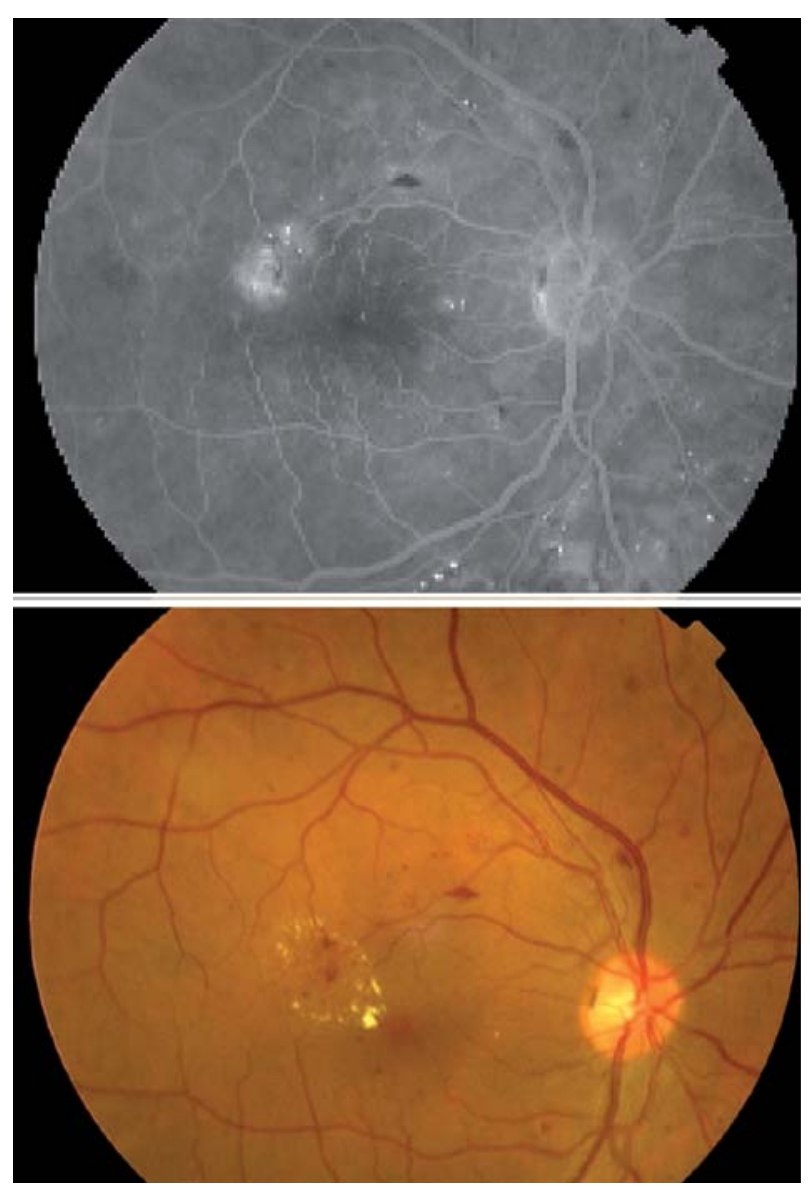

Fig. 4: EMCS focal. 
como EM cuando existían exudados duros dentro de 1 DD del centro.

- Con Edema Macular Clínicamente Significativo (EMCS):

- Engrosamiento retiniano dentro de $500 \mu \mathrm{m}$ del centro (fig. 1).

- Exudados duros dentro de $500 \mu \mathrm{m}$ del centro si se asocia a un engrosamiento de retina adyacente (fig. 2).

- Un área de engrosamiento retiniano del tamaño de al menos 1 área del disco, parte de la cual está a menos de $1 \mathrm{DD}$ del centro (fig. 3).

La mejor agudeza visual corregida (MAVC) no forma parte de la definición de edema macular y ésta puede ir de 1,2 a percepción de luz (PL), ambas incluidas, y presentar EMCS.

\subsection{Clasificación Angiográfica del EM}

- EM focal: Área bien definida de difusión procedente de microaneurismas individuales o asocia- dos. Del mismo modo se ha definido como EM focal aquel con un área de difusión en la que $\geq 67 \%$ está asociada a microaneurismas (4,5). Su imagen fundoscópica característica es de un anillo circinado que amenaza o compromete el centro de la mácula (fig. 4).

- EM multifocal: Con varias áreas de difusión (que frecuentemente se confunde con el EM difuso) (fig. 5).

- EM difuso: Se ha definido como un área de hiperfluorescencia de difusión tardía $\geq 2$ DD con afectación foveal (6-11) o como aquella hiperfluorescencia de la cual $\leq 33 \%$ está asociada a microaneurismas (3). Suele ser bilateral y asimétrico. Clínicamente se traduce por un área de edema poco definida, con escasos microaneurismas y pocos exudados duros. En ocasiones se aprecian brillos y opacidad de la hialoides posterior, con o sin membranas epirretinianas. Se asocia con frecuencia al edema macular quístico (EMQ) (fig. 6).
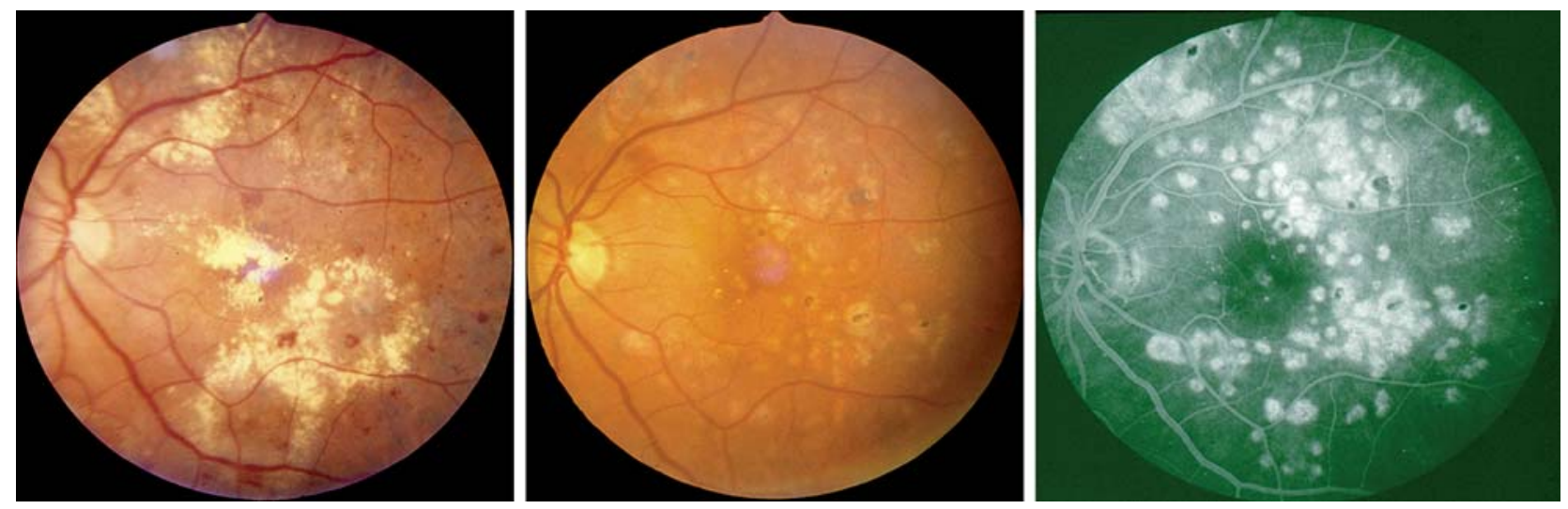

Fig. 5: EMCS multifocal antes y después del tratamiento con láser.
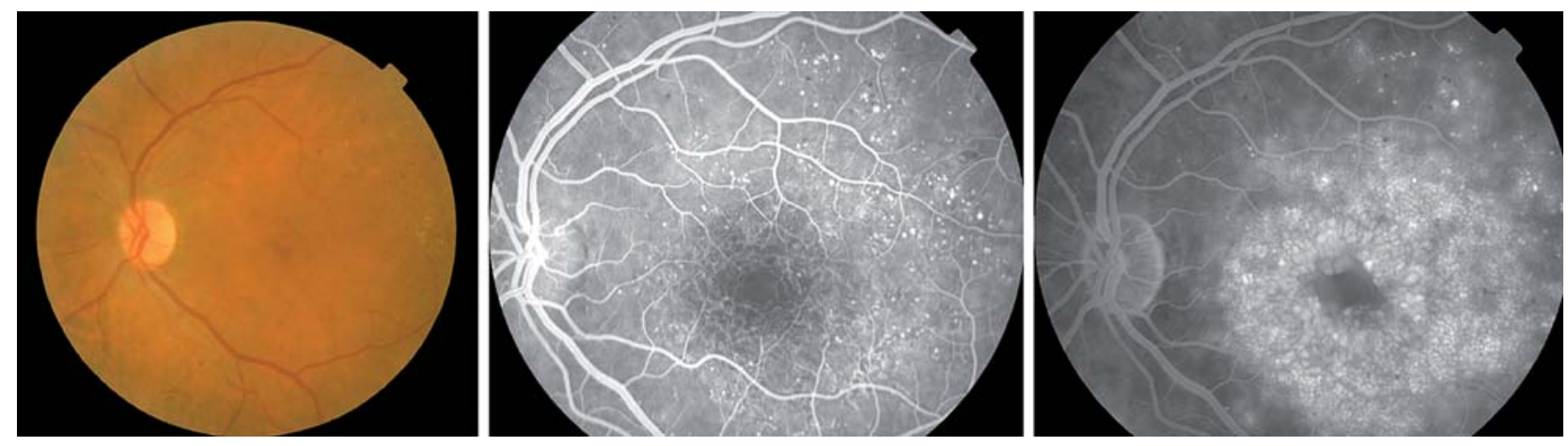

Fig. 6: EMCS difuso. 

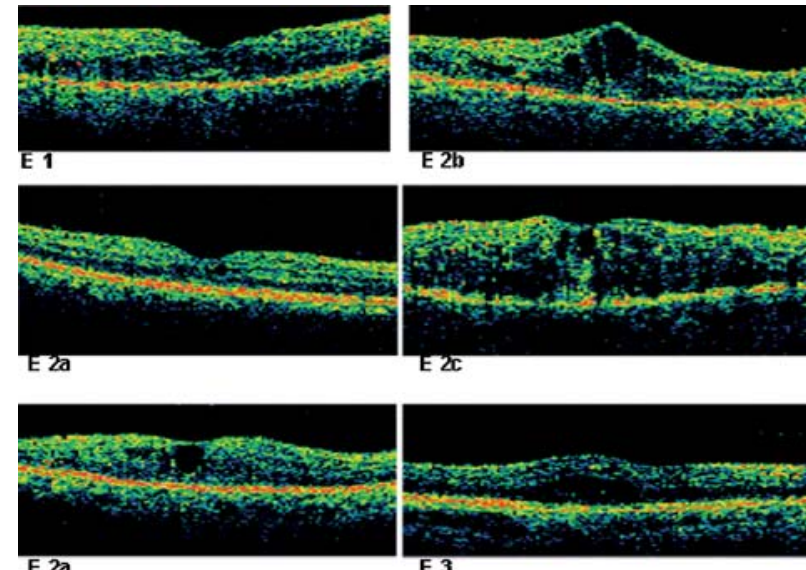

Fig. 7a: Clasificación del EM según su morfología en OCT.

- EM mixto: Ambos tipos de edema, focal y difuso, pueden coexistir dando lugar al llamado edema macular de tipo mixto.

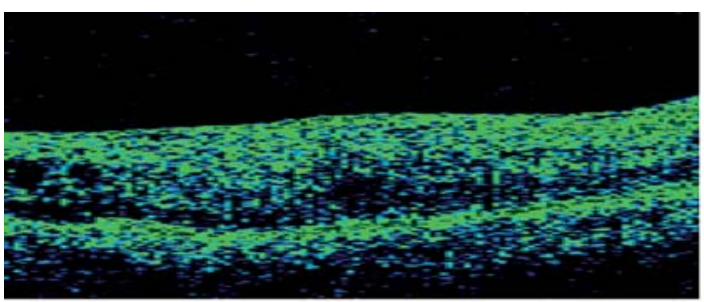

T1

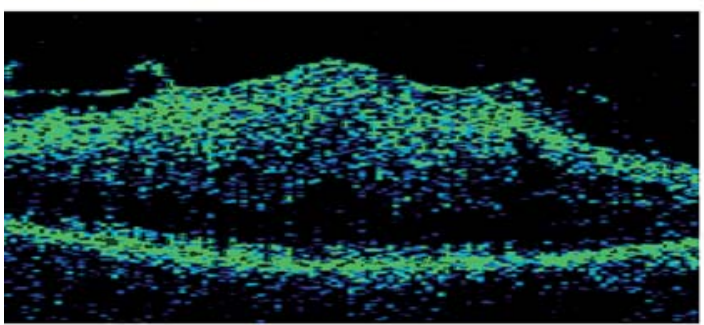

T 2

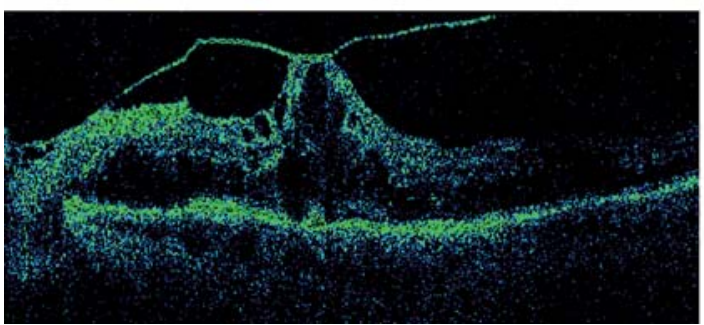

T 3

Fig. 7b: Clasificación del EM según la existencia de tracción epirretiniana en OCT.

\subsection{Clasificacion basada en la Tomografía de Coherencia Óptica (OCT)}

La clasificación del EM basada en el OCT (12) se divide en:

1. Atendiendo a la morfología (fig. 7a):

E1: Engrosamiento simple (en esponja, que afecta a las capas más externas de la retina y en donde no se observan espacios cistoides).

E2: Engrosamiento cistoide. Engrosamiento de la retina que se asocia con quistes aumentando de severidad de «a» (de 2 a 4 pequeños quistes) hasta «c» (coalescencia de varios quistes).

E3: Desprendimiento del neuroepitelio. Puede presentarse aislado o asociado a engrosamiento retiniano simple o quístico.

2. Atendiendo a la existencia de tracción epirretiniana (fig. 7b).

(Existencia de una línea continua hiperreflectante sobre la superficie interna de la retina, con al menos un punto de adhesión a la retina en al menos 1 de los 6 cortes del mapa retiniano.)

TO: Ausencia de la línea hiperreflectante.

T1: Presencia de la línea continua hiperreflectante pegada a la retina pero sin distorsionarla.

T2: La línea continua hiperreflectante tiene múltiples puntos de unión a la retina y la distorsiona.

T3: Tracción anteroposterior con la configuración típica en «alas de gaviota».

\section{MANEJO DE LA RETINOPATÍA DIABÉTICA}

\section{Establecimiento de la primera exploración oftálmica}

- Diabetes tipo 1: la primera revisión oftálmica se hará a los 3-5 años del diagnóstico de la DM. En caso de presentarse algún signo de retinopatía se aconseja mantener el control ocular en los centros hospitalarios, en base al alto grado de complicaciones y el carácter agresivo de las mismas.

- Diabetes tipo 2: la primera revisión se hará en el momento del diagnóstico aconsejándose controles anuales o bianuales posteriores si no hay factores de riesgo asociados hasta la aparición de algún grado de retinopatía. Al detectarse ésta, los criterios aconsejados son expuestos según el grado de afectación, la existencia o no de edema, y/o la presencia de complicaciones secundarias a 
una retinopatía avanzada. Se debe prestar especial atención a aquellos pacientes diabéticos tipo 2 de aparición temprana, y más concretamente entre los 30 y 50 años, debido a su mayor expectativa de vida y un control metabólico habitualmente inadecuado, así como aquellos en tratamiento con insulina ya que, en relación con las complicaciones, se pueden comportar como diabéticos tipo 1 (13).

\section{Seguimiento}

Para establecer la frecuencia de las revisiones posteriores se tendrá en cuenta, por un lado, el grado de retinopatía y, por otro, el estado de la mácula. En cuanto a la periodicidad de las revisiones primará el criterio de mayor riesgo visual. Por ejemplo si un paciente con RDNP leve, cuyo control aconsejado es anual, presenta también un EMCS asociado, la periodicidad de las visitas las dictará este último (cada 3 meses).

En todos los estadios de la RD es esencial el control endocrinológico fundamentalmente de: glucemia, hemoglobina glicosilada, microalbuminuria en orina, hipertensión arterial (HTA), sobrepeso, lípidos, estado cardíaco y renal. Esto tiene consecuencias en dos sentidos, por un lado si existe un desarreglo metabólico importante nuestro control ocular deberá ser más frecuente y, por otro lado, podemos posponer el tratamiento ocular, por ejemplo de un edema macular, hasta que se consigan niveles aceptables de glucemia y/o TA. Asimismo se recomendará al paciente dejar de fumar.

\subsection{Sin Retinopatía Diabética Aparente}

Examen oftálmico bianual si existe buen control metabólico ( $\mathrm{Hb} \mathrm{A} 1 \mathrm{c}<7 \%)$ y ausencia de factores de riesgo asociados (HTA, dislipemias, etc.). En estos casos la exploración oftálmica bianual (14) parece ser suficiente para detectar a tiempo tanto el edema macular como la RDP. Esto reduciría el número de primeras visitas al oftalmólogo en un $25 \%$ lo cual disminuiría considerablemente los costes sanitarios y evitaría al diabético algunas exploraciones innecesarias.

Exploraciones oftalmológicas anuales si existen factores de riesgo asociados o el control metabólico es dudoso o deficiente.

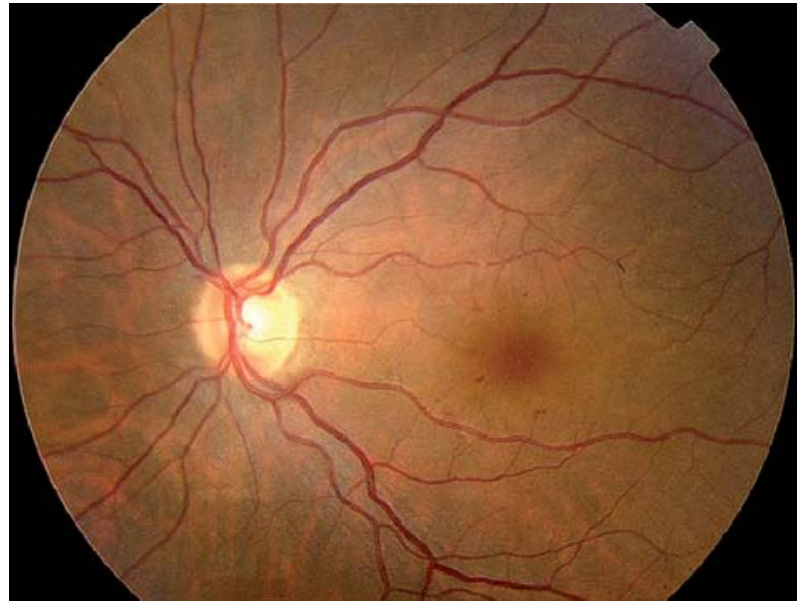

Fig. 8: RDNP leve.

\subsection{Con RDNP Leve}

Control oftalmológico anual.

Adelantar el control en caso de: gran cantidad de $\mu \mathrm{A}$ o que éstos amenacen fóvea, primera exploración de un paciente mal controlado, paso reciente de antidiabéticos orales a insulina, pacientes embarazadas (se hará un control en cada trimestre y luego cada 6 meses durante el primer año tras el parto).

En este estadio es fundamental informar y concienciar al paciente sobre la importancia del control metabólico. Según el DCCT (Diabetes Control and Complications Trial) (15-18) en los diabéticos tipo 1 que mantienen un nivel medio de $\mathrm{Hb} \mathrm{A} 1 \mathrm{c}$ del

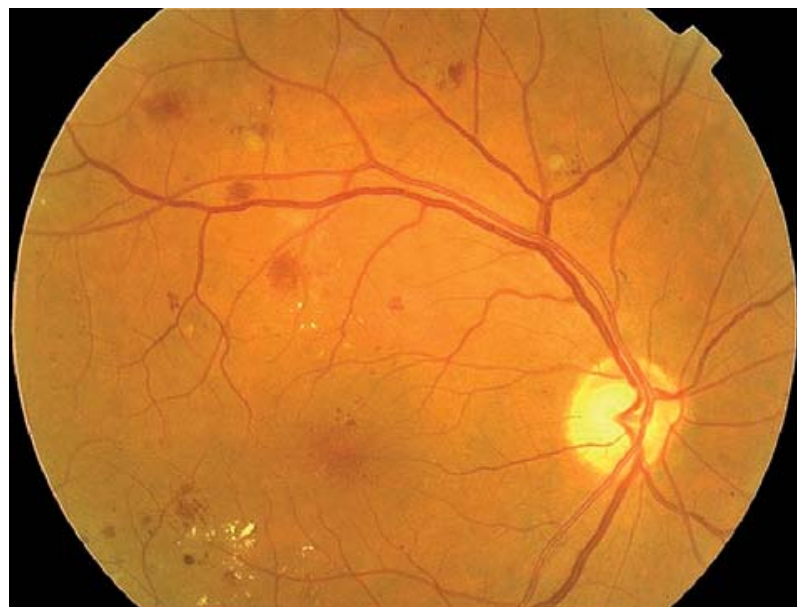

Fig. 9: RDNP moderada. 
$7,2 \%$ se reduce la incidencia de la retinopatía en un $76 \%$ y la progresión de la retinopatía diabética en un $54 \%$.

El UKPDS (United Kingdom Prospective Diabetes Study) (19) constató hallazgos similares en los diabéticos tipo 2. Además demostró que el control estricto de la tensión arterial disminuye la progresión de la RD en un 34\% y el deterioro de la agudeza visual en un $47 \%$ (20) (fig. 8).

\subsection{Con RDNP Moderada}

Control oftalmológico semestral.

No precisa AFG. No precisa panfotocoagulación (PFC) dado que el riesgo de progresión a RDP al año oscila entre un $5,4 \%$ y un $26,3 \%$. Hay que tener en cuenta que en este epígrafe se recoge una gran variabilidad de cuadros, desde aquellos que sólo presentan $\mu \mathrm{A}$ con un exudado duro hasta otros más abigarrados en que éstos se acompañan de «exudados» algodonosos, hemorragias intrarretinianas e incluso algún arrosariamiento venoso por lo queda a criterio del oftalmólogo el tiempo exacto de seguimiento (fig. 9).

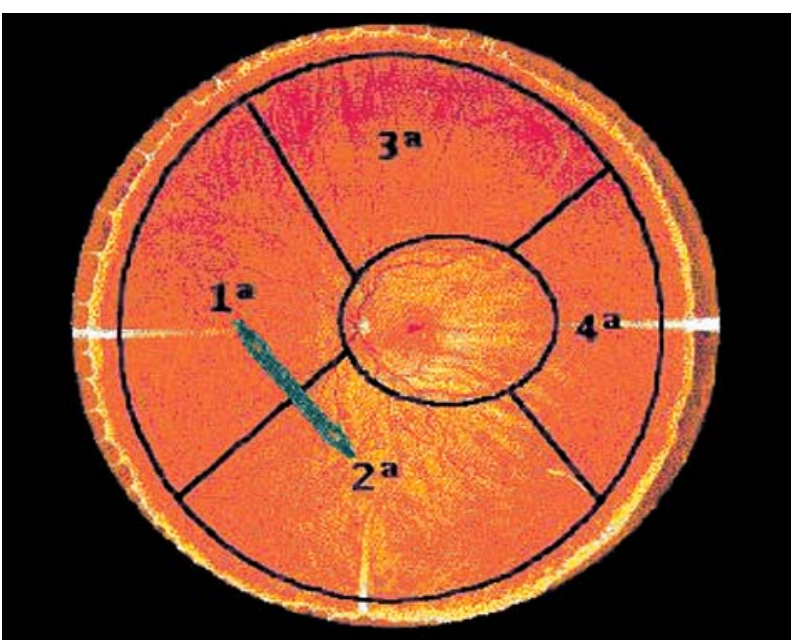

Fig. 10: Secuencia de Fotocoagulación por cuadrantes.

\subsection{Con RDNP Severa}

Las RDNP severas son casos que deben ser analizados con cautela, dada la probabilidad de progresión a RDP que se sitúa entorno al 50,2\% en un año [un 14,6\% con características de alto riesgo (21)].

- DM 2: Control hospitalario cada 3 - 4 meses.

- DM 1 con mal control metabólico: revisión hospitalaria cada 2 meses.

Considerar PFC temprana en aquellos pacientes con mayor riesgo de progresar: DM 2 con mal control metabólico, pacientes que no cumplen las revisiones de forma habitual, RDP en el otro ojo, pacientes con cataratas con significación visual evidente que pueda limitar en un futuro próximo la PFC, previo a la cirugía de catarata, embarazo o intención de lograrlo y áreas de isquemia angiográfica generalizadas.

En estos casos el orden de fotocoagulación que se aconseja es realizar un cuadrante por sesión comenzando por el nasal o el inferior y finalizando por el temporal (fig. 10). En cuanto a la frecuencia, se dará una sesión cada 2-3 semanas (si se puede esperar, mejor cada 3 semanas) ya que la mácula no se recupera en 1 sola semana (22). Antes de comenzar

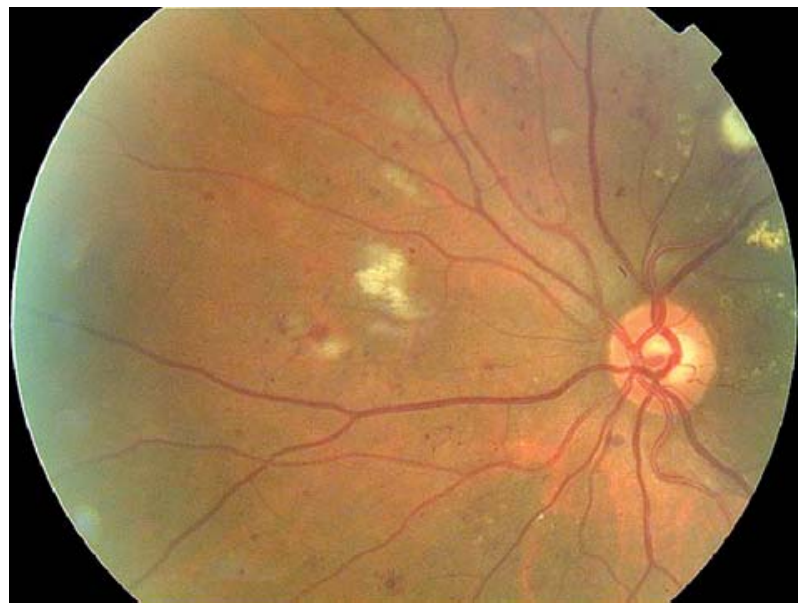

Fig. 11: RDNP severa.

Tabla II. Grados de magnificación de lentes

Factores de magnificación de las lentes de contacto que no inducen un incremento significativo del tamaño de la quemadura del láser

Factores de magnificación de las lentes de contacto que sí inducen un incremento significativo del tamaño de la quemadura del láser
Mainster focal gird (1.05), Volk area centralis (1.0) y la lente Goldmann de 3 espejos (1.08)

Cuadrasférica (1.92), Supercuadrant 160 (2) y Ultrafield (1.89). Inducen una doble magnificación del tamaño del spot, por lo que el tamaño de la quemadura del láser debe reducirse a la mitad 
la fotocoagulación debe tenerse en cuenta los diferentes grados de magnificación de las distintas lentes disponibles (tabla II).

Existen algunos trabajos que proponen el uso de antiVEGF como coadyuvantes de la PFC, en un intento por minimizar el edema macular post-láser (23-25).

Es importante enfatizar que en los casos con RDNP severa que hayamos decidido realizar una PFC y que además presenten edema macular clínicamente significativo se tratará primero éste y posteriormente se hará la PFC (fig. 11).

\subsection{RDP y Medios Transparentes}

PFC en un mes o mes y medio: sesiones de 300500 impactos (si existe tracción hacer sesiones de 200-300 impactos) iniciando por el cuadrante inferior. Revisiones cada 3-6 meses. Cuando se propone realizar una PFC es aconsejable explicar al paciente los siguientes puntos:

a. La evidencia científica demuestra que la PFC puede detener la progresión de la RDP, pero no en todos los casos.

b. El riesgo de hemorragia persiste después de la PFC, porque la regresión de la neovascularización es lenta.

c. La PFC puede producir disminución moderada de visión, del campo visual o de la adaptación a la oscuridad, pero el beneficio supera con creces los efectos colaterales.

Es conveniente insistir al paciente acerca de estos puntos haciendo hincapié en que de producirse una hemorragia vítrea ésta es consecuencia de su enfermedad y no del tratamiento con láser.

Si no responde (avanza la proliferación, se producen sangrados...) se debe revisar el nivel de PFC valorando que tanto la confluencia como la extensión hacia periferia extrema sean los adecuados. Una vez comprobado que la PFC es correcta tenemos dos opciones: 1. AntiVEGF (El empleo tanto de triamcinolona (TAIV) como de antiVEGF en la retinopatía diabética está en fase de ensayo clínico. $\mathrm{Su}$ uso, fuera de ensayo clínico, debe contar con el consentimiento informado por escrito del paciente, advirtiendo de la falta de experiencia a largo plazo y de las posibles complicaciones, y con la autorización de la Agencia Española del Medicamento (uso compasivo). La solicitud debe dirigirse a la Dirección General de Farmacia y Productos Sanitarios del

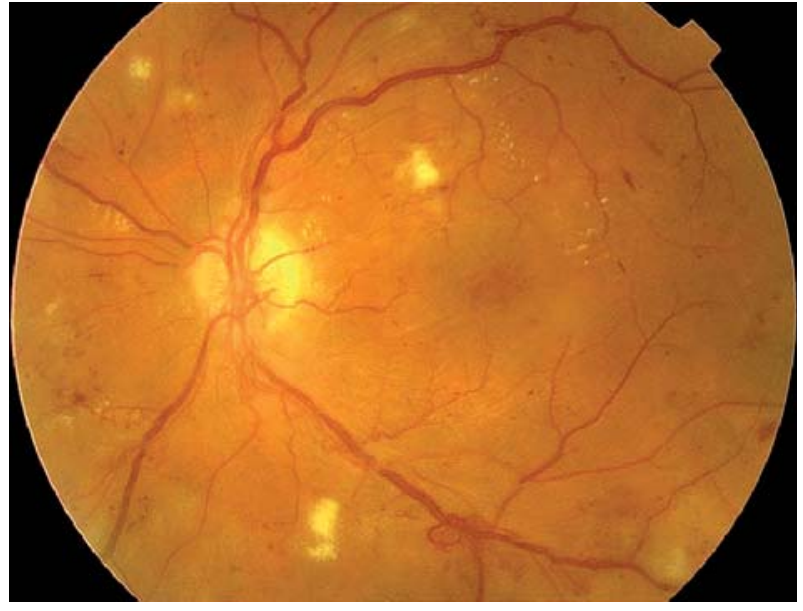

Fig. 12: RDP.

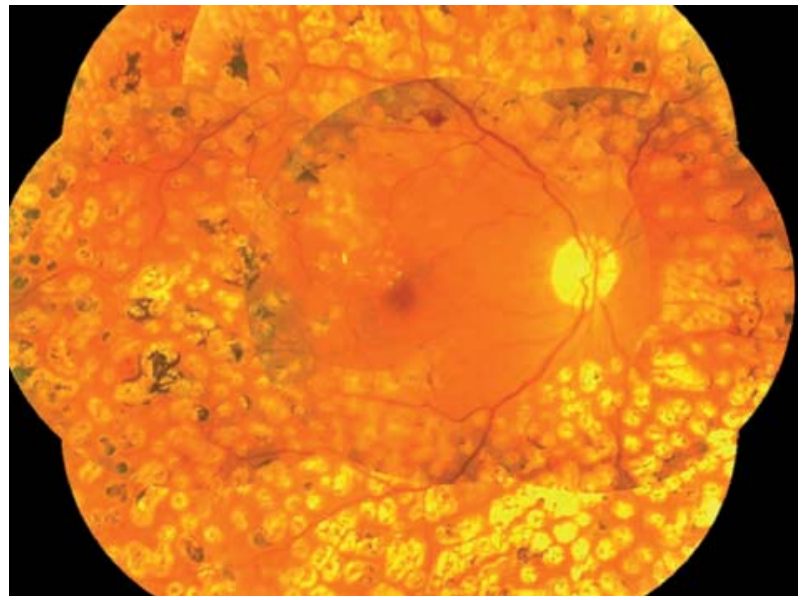

Fig. 13: RDP después de PFC.

Ministerio de Sanidad y Consumo): cada 4-6 semanas. 2. Cirugía retinovítrea (CRV) (figs. 12 y 13).

\section{Casos Especiales}

- Con adherencias vitreorretinianas extensas no activas, sin tracción y sin amenaza del área macular y buena agudeza visual : revisiones periódicas.

- Con adherencias vitreorretinianas extensas que amenacen mácula : CRV con PFC. Se puede usar antiVEGF + CRV antes del 3. ${ }^{\text {er }}$ día \pm antiVEGF al final de la cirugía.

- RDP con tracciones activas pero sin hemovítreo severo : antiVEGF previo a la cirugía: 1 ó 2 días antes para minimizar sangrado perquirúrgico.

- Papilopatía diabética traccional : CRV. 
- Maculopatía traccional : CRV.

- RDP + rubeosis con transparencia de medios : $\mathrm{PFC}$ inmediata.

- RDP + rubeosis + glaucoma neovascular : PFC inmediata \pm antiVEGF + válvula.

Tras la inyección intravítrea de bevacizumab (IVB) se ha descrito (26) una rápida contracción de la membrana neovascular que tracciona de la raíz del iris produciéndose un cierre angular sinequial permanente. Esta situación indeseable puede prevenirse evitando el uso de antiVEGF intravítreo en ojos que presenten amplias zonas de sinequias anteriores periféricas. La exploración gonioscópica se hace imprescindible puesto que la afectación del ángulo puede preceder a la del iris y pasar ésta desapercibida con sólo la exploración biomicroscópica. La ablación de la retina isquémica mediante PFC con láser, o crioablación -cuando el láser no sea posible- permite lograr el mismo efecto que los antiVEGF de forma más duradera.

\subsection{RDP con Hemovítreo}

Si el fondo de ojo es inexplorable, la ecografía ocular es obligada.

Limitar la actividad de los pacientes diabéticos con RDP no es eficaz en la prevención del hemovítreo. De existir el sangrado, tampoco prescribir reposo resulta eficaz. Por ello, ante los dos supuestos, el paciente puede realizar la actividad que le permita su limitada visión.

- Si DR regmatógeno o mixto (traccional/regmatógeno) : CRV urgente.

- En paciente no panfotocoagulado : CRV preferente.

- En paciente panfotocoagulado y sin DR : Esperar 2 meses. Controles de AV/Ecográficos cada 15 días. Cirugía diferida (2-3 meses) de no haber mejoría. En esos casos es útil valorar el estado del ojo contralateral.

Algunos autores (27-29) recomiendan considerar el uso de antiVEGF en caso de hemorragia persistente o muy severa una vez descartada la presencia de un DR traccional. Si el paciente no está vitrectomizado, se puede valorar el uso de terapia antiangiogénica previa a la cirugía, siendo aconsejable que ésta se realice entre el $1 .{ }^{\text {er }}$ y $3{ }^{\text {er }}$ día previos a la intervención. Si el paciente está ya vitrectomizado, la duración de la acción del antiVEGF se acorta considerablemente. Se podrían inyectar cada 4 semanas manteniendo una estrecha vigilancia por riesgo de desprendimiento de retina traccional. Es importante recordar que el empleo de antiVEGF se hace bajo uso compasivo.

\section{Casos Especiales}

RDP + rubeosis + opacidad de medios:

- Si no existen sinequias anteriores periféricas (la presencia de sinequias anteriores periféricas a nivel angular cuestiona el uso de los antiVEGF, dado que la contracción severa del tejido como respuesta de la inyección intravítrea puede inducir el cierre angular agudo y el desarrollo de un glaucoma neovascular de nefastas consecuencias. Por ello, en el caso de paciente con RDP donde se plantee la opción terapéutica de antiVEGF, debe tener como requisito la gonioscopia angular) : antiVEGF + cirugía para resolver la opacidad de medios y tratar la RDP

- Si existen sinequias anteriores periféricas : cirugía para tratar la opacidad de medios y la RDP. En caso de que evolucionara a glaucoma neovascular: Implante de Válvula.

\subsection{RDP Con Hemorragia Premacular- Retrohialoidea}

CRV precoz, preferiblemente antes de un mes.

\subsection{RDP Con Hemovítreo Recurrente tras CRV}

\section{Sangrado inmediato (en el primer mes)}

Ocurre a partir de restos de tejido fibrovascular disecado durante la CRV o dispersión de sangre residual (30), aunque pudiera deberse también a la existencia de DR o desgarros periféricos, a veces, en relación con las esclerotomías. Ambas posibilidades deben descartarse.

La gran mayoría de los sangrados inmediatos se resuelven espontáneamente en días o semanas. Esperar 1-2 meses con control de AV y ecográfico. Si no desaparece existen dos opciones:

- Recambio fluido-aire y en caso de no mejoría realizar CRV.

- CRV directamente.

La cirugía busca restablecer la visión y eliminar los productos que puedan estimular la proliferación. 
De existir un hemovítreo recurrente se aconseja control ecográfico cada 15 días. Tenemos dos opciones:

- Nueva CRV en 2-4 meses. (antiVEGF pre o intra-operatorio).

- AntiVEGF cada 4-6 semanas.

Si mejora: completar PFC/ crioterapia en periferia extrema.

\section{Sangrado tardio (después del primer mes)}

Aparece en un número significativo de casos (del $13 \%$ al $50 \%$ ), y puede tener distintas causas. Exponemos las dos más frecuentes.

- Sangrado del tejido proliferativo periférico presente en las esclerotomías: el origen de estos neovasos no es la retina anterior (como en la proliferación fibrovascular anterior) sino el cuerpo ciliar. Este tejido fibrovascular se puede extender hacia vítreo anterior dada su proximidad. Se asocia a incarceración del vítreo en las esclerotomías. El diagnóstico puede hacerse por depresión escleral. A veces puede existir un vaso centinela epiescleral entrando en la esclerotomía (aunque su existencia no asegura que exista tejido fibroproliferativo en la misma).

El tratamiento consistirá en una nueva CRV extrayendo este tejido, pudiéndose dejar gas como tamponador.

Recientemente se ha propuesto el uso de antiVEGF en hemorragias vítreas recurrentes tardías (31).

- Proliferación fibrovascular anterior (32). En estos casos, el hemovítreo ocurre entre el $1 .^{\text {er }}$ y $7 .^{\circ}$ mes después de la cirugía retinovítrea. Se trata de una proliferación neovascular que se origina en la retina periférica y se extiende a través de la hialoides anterior hacia la cara posterior de la cápsula cristaliniana. El examen con depresión escleral y la biomicroscopía indirecta revelan la neovascularización, a veces aparece también rubeosis en iris. En la ecografía pueden detectarse bandas de tejido engrosado desde la retina periférica al cuerpo ciliar y superficie posterior del iris, desprendimientos traccionales de la retina anterior y del cuerpo ciliar (que se asocian con frecuencia a hipotonía). Como su tratamiento es desalentador, lo más importante es la prevención, sobre todo, en pacientes con factores de riesgo de padecerla como: jóvenes con DM tipo 1, isquemia retiniana severa, DR traccional y/o mixto, sobre todo si se les ha colocado cerclaje extraescle- ral, múltiples cirugías, RDP no tratada o que no responde a la PFC, RDP con proliferación posterior fibrovascular extensa, rubeosis iridis postoperatorias, hemovítreos recurrentes...

En éstos hay que hacer PFC agresivas. Si aún así evolucionan, lo indicado es una CRV extrayendo cuidadosamente el vítreo anterior (para lo que puede ser necesaria una lensectomía) y realizar una fotocoagulación anterior extensa.

Si la proliferación fibrovascular anterior ya está presente hay que detectarla lo más precozmente posible, antes de que ocurra un desprendimiento traccional de retina y/o de cuerpo ciliar y se formen membranas ciclíticas. Se debe hacer una CRV seguida de lensectomía extrayendo la cápsula posterior del cristalino, disección de la base del vítreo, endodiatermia de neovasos y endofotocoagulación lo más extensa posible (evitar la crioterapia ya que ésta puede causar contracción del tejido fibrótico y DR periférico). Si existen desprendimientos traccionales periféricos de retina y/o del cuerpo ciliar con frecuencia se necesita hacer retinotomías periféricas y usar aceite de silicona para conservar el globo ocular.

\subsection{RDP con Desprendimiento de Retina}

Traccional que amenace o afecte la mácula $=$ CRV con o sin el empleo de TAIV como manipulador.

Regmatógeno/mixto: CRV $<48 \mathrm{hr}+$ gas de larga duración o aceite de silicona.

\subsection{RDP con Isquemia Anterior}

Ante un cuadro de rubeosis y de glaucoma neovascular es importante el estudio del ángulo camerular. Como ya se ha expuesto previamente, este requisito es fundamental en casos en los que se plantee el uso de antiVEGF.

\subsection{RDP con Rubeosis}

Incipiente : completar la PFC y vigilancia a los 2 meses.

Evidente : completar la PFC, terapia antiangiogénica, vigilancia al mes.

En caso de una rubeosis de iris con catarata que impide la fotocoagulación: podríamos plantearnos 
el uso de antiVEGF. Éstos se inyectarán 2 ó 3 días previos a la cirugía de la catarata, una vez descartada la presencia de neovasos angulares, y posteriormente se realizará la PFC.

Si la rubeosis de iris se acompaña de hemorragia vítrea que impide la fotocoagulación : antiVEGF seguidos de CRV (a los 2 ó 3 días después de la inyección), intentando ampliar la PFC hasta extrema periferia.

\subsection{Glaucoma Neovascular (GN)}

El GN es un tipo de glaucoma secundario resultante de la obstrucción de la red trabecular por elementos fibrovasculares y/o sinequias asociadas.

Suele cursar con dolor ocular, disminución severa de la agudeza visual, presiones intraoculares muy altas, hiperemia conjuntival, edema corneal, tyndall, hiphema, rubeosis de iris, corectopia, ectropion uveal y goniosinequias importantes.

Es probable que en el futuro inmediato veamos cambiar el pronóstico de esta enfermedad gracias a los antiVEGF. Aunque los resultados parecen ser muy esperanzadores a corto plazo, actualmente no disponemos de estudios prospectivos que nos informen sobre las dosis de elección, los efectos secundarios y la seguridad a largo plazo, ni cuál es la mejor combinación con otras medidas terapéuticas.

En todos los enfermos con riesgo se debe hacer un examen ocular completo que incluya: exploración sin midriasis del borde pupilar con gran aumento y gonioscopia.

El tratamiento del GN tiene tres frentes terapéuticos (33-35).

\section{Tratamiento Médico}

- Hipotensores oculares tópicos: betabloqueantes, alfa-adrenérgicos, inhibidores de la anhidrasa carbónica. La pilocarpina está contraindicada y los análogos de las prostaglandinas son discutibles (está bloqueado el acceso a la vía uveoescleral por el tejido fibrovascular en el ángulo y aumenta la inflamación).

- Hipotensores oculares sistémicos: diuréticos osmóticos e inhibidores de la anhidrasa carbónica intravenosa y oral.

- Controlar el componente inflamatorio, que siempre está presente y es muy intenso, con midriáticos (Atropina o Ciclopléjico) y corticoides tópicos (Dexametasona o metil prednisolona/3 horas). El efecto de esta medida, no sólo controla la inflama- ción, sino que disminuye el dolor y disminuye la presión intraocular. Si es preciso, corticoides subconjuntivales o subtenonianos.

\section{Tratamiento Anti-vasoproliferativo}

Está indicada la PFC (evidencia de nivel A): con 1.200-1.600 impactos se produce la regresión de la rubeosis en el 70,4\% de los casos. La PFC destruye la retina isquémica responsable de la síntesis de factores vasoproliferativos, y además aumenta la disponibilidad de oxígeno en la retina no tratada. La PFC se hará con carácter urgente y siempre completa hasta extrema periferia. Sin embargo, la regresión de los neovasos después de la PFC es lenta (semanas), por lo que de forma complementaria, podría plantearse el uso de antiVEGF (36) que provoca una regresión de la rubeosis casi inmediata, de 2448 horas. Estos ojos deben ser controlados de forma cercana ya que, como hemos hecho referencia anteriormente, el antiVEGF podría provocar la contracción del tejido fibrovascular existente generando un cierre angular. En cualquier caso se debe exigir al paciente un control estricto de las glucemias. Esto es así dado que la cifra de Hb A1c es el factor más importante en la recurrencia de una RDP una vez PFC.

Si el diagnóstico ha sido precoz, es posible que con estas medidas se consiga solucionar el cuadro.

\section{Tratamiento quirúrgico}

Siempre que sea posible debe intentar posponerse hasta que desaparezca la rubeosis.

- Trabeculectomía con antimetabolitos.

- Implante valvular.

- Ciclofotocoagulacón (37) con láser de diodo.

No existe consenso en cuanto a cuál de estas técnicas es la más indicada. La cirugía filtrante, con o sin válvula de drenaje, parece ser la mejor opción si la retina está panfotocoagulada, la rubeosis ha regresado y existe un bloqueo angular por las sinequias periféricas. El uso de antiVEGF combinado con la cirugía filtrante, puede ayudar a disminuir los sangrados en vítreo y la cicatrización de la ampolla de filtración, sobre todo en los casos de rubeosis aún activa. Se ha usado el bevacizumab como tratamiento coadyuvante a la cirugía filtrante con buenos resultados $(38,39)$.

En cuanto a los procesos ciclodestructivos, el de elección es el realizado con láser de diodo. El efec- 
to es, a veces, transitorio pero suele ser suficiente para completar la PFC.

El procedimiento es bastante seguro en casos de glaucomas refractarios, pero es en el glaucoma neovascular donde parece tener una mayor incidencia de hipotonías postratamiento $(15 \%)$.

Resumiendo lo anteriormente expuesto, la pauta de tratamiento para el GN que aconsejamos está en consonancia con la recientemente editada por la Sociedad Europea de Glaucoma (40) y es:

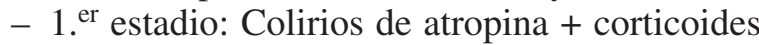
tópicos + antiglaucomatosos: todos excepto pilocarpina. Secuencia sugerida: Brimonidina, betabloqueantes, inhibidores de anhidrasa carbónica: tópicos o sistémicos. PFC: completarla al máximo. La terapia antiangiogénica ha demostrado ser útil pero aún no tiene aprobado su uso para esta indicación.

$\neq 2$. $^{\circ}$ estadio: Sin respuesta en la PIO. Trabeculectomía con antimetabolitos / implantes valvulares / cicloablación. Fenolización (si ojo doloroso en amaurosis) / evisceración / enucleación.

\subsection{Catarata}

Los diabéticos $<65$ años tienen entre 3 y 4 veces mayor riesgo de desarrollar catarata que la población no diabética (en los grupos más jóvenes el riesgo aumenta hasta 25 veces). A partir de los 65 años el riesgo se iguala.

Siempre que la catarata permita visualizar el fondo ocular y tratar con láser la progresión de la retinopatía, la postura será expectante. Cuando se superen estos límites, la facoemulsificación esta indicada.

Tras la cirugía, el paciente debe ser fotocoagulado si procede o vigilado (6 meses) por si el trauma quirúrgico induzca la progresión de su retinopatía. Hay que tener en cuenta que en los diabéticos sin EM que se sometan a una cirugía de catarata, puede aparecer un aumento del grosor macular central en los primeros 3 meses de la cirugía, aunque posteriormente algunos se resolverán de forma espontánea (41).

En los diabéticos que ya tenían EM éste debe ser tratado previo a la cirugía de la catarata si es que ésta lo permite. Si la catarata impidiera el tratamiento del EM puede plantearse el uso de antiVEGF/TAIV peroperatorios. Actualmente hay ensayos clínicos en marcha para evaluar su eficacia $(42,43)$.

Resumiendo, ante una RD sin EM deben establecerse controles periódicos tras la cirugía de la catarata. Si aparece un EM mantendremos una actitud expectante tratando de descartar que se trate de un EM pseudofáquico, cuya evolución espontánea suele ser favorable. A veces, tanto por el aspecto clínico como angiográfico, puede resultar muy difícil distinguir si estamos ante un EM diabético o ante un Sd. de Irvine-Gass. No obstante, si el edema se mantiene en el tiempo, lo trataríamos según el esquema propuesto en esta guía.

Dado el riesgo de progresión de la RD, se desaconseja la cirugía de catarata de ambos ojos de forma simultánea o diferida con poco tiempo de intervalo entre ellas.

Es importante recalcar que, como en todo paciente con patología macular, en el EMD no es aconsejable la implantación de Lentes Intraoculares multifocales o difractivas (gráfico 1).

\section{MANEJO DEL EDEMA MACULAR DIABÉTICO}

A tal fin, y como ya se ha señalado anteriormente, se utilizará la clasificación del EDTRS y se planteará tratamiento siempre que exista un EMCS. Del mismo modo será crítico el resultado del OCT para decidir el tratamiento de elección.

\subsection{Valoración previa a la actuación terapéutica}

Si en la retinopatía diabética, el control sistémico es indiscutible, en el edema macular esto resulta aún más evidente. El abordaje de esta complicación debe ser multidisciplinar. Es preciso insistir en el control de la glucemia (HbA1C), presión arterial, sobrepeso y lípidos enviando al paciente al endocrino/internista/nefrólogo siempre que sea necesario dada la gran influencia de estos factores de riesgo. Un mal control metabólico puede, en algunos casos, justificar posponer el tratamiento del edema macular hasta que éste mejore, siendo deseable que las cifras de $\mathrm{HbA1C}$ se situaran por debajo de 7,5\%.

La valoración del edema debe incluir siempre: mejor agudeza visual corregida, examen biomicroscópico, retinografía y OCT. La AFG puede considerarse discutible en algunos casos claros de circinadas donde se ve el origen de la exudación. Sin embargo, es una prueba muy útil para estudiar el estado de la red vascular perifoveloar. 
Control endocrinológico: Es especialmente importante el control de glucemia $(\mathrm{HbA} 1 \mathrm{c}<7 \%)$, HTA $(<130 / 80$ ), lípidos ( $T \mathrm{G}<150 \mathrm{mg} / \mathrm{dl}$; LDL colesterol $<100 \mathrm{mg} / \mathrm{dl}$ salvo en pacientes con alto riesgo cardiovascular que debe ser $<70 \mathrm{mg} / \mathrm{dll}$ ). Estado cardíaco y renal. Evitar el sobrepeso. Ejercicio físico.

Paciente que no cumple estos valores: enviarlos al endocrino/internista/médico de familia.

\section{SIN RD APARENTE}

Control oftalmológico anual o bianual.

\section{RDNP LEVE}

Control oftalmológico anual.

Adelantar control en caso de: gran cantidad de $\mu \mathrm{A}$, que almacenen fóvea..., primera exploración de paciente mal controlado, paso reciente de antidiabéticos orales a insulina, adolescentes, embarazadas ...

\section{RDNP MODERADA}

Control oftalmológico semestral.

\section{RDNP SEVERA}

Revisión cada 2-4 meses.

Considerar PFC temprana en caso de: DM2 con mal control metabólico y pacientes que no cumplen las revisiones de forma habitual, RDP en el ojo, pacientes con cataratas con significación visual evidente que pueda limitar en un futuro próximo la PFC, previo a la cirugía de la catarata, embarazo o deseo del mismo, isquemias AFG generalizadas ...

\section{RDP y medios transparentes:}

PFC 1-11/2 mes: sesiones de 300-500 impactos (si existe tracción, hacer sesiones de 200-300 impactos). Revisiones cada 3-6 meses.

Orden de fotocoagulación: según gráfica. Frecuencia: sesiones cada 2-3 semanas (si se puede esperar, mejor cada 3 semanas). La mácula no se recupera en 1 sola semana.

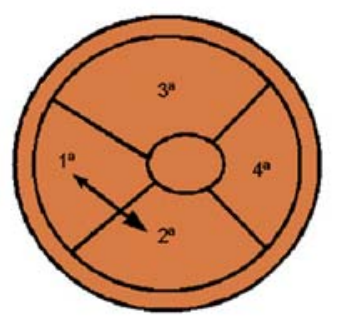

Si no responde: Opciones: $\quad 2$

1. AntiVEGF: cada $4-6$ semanas

(1)

1. AntivEGF: cada 46 semanas

2. Si no responde: CRV.

\section{RDP con HEMOVÍTERO:}

- Si DR regmatógeno o mixto: CRV urgente.

- Si no DR: esperar 2 meses con controles de AV/Ecográficos en 2-4 semanas.

De no haber mejoría se hará cirugía diferida (2-3 meses).

Gráfico 1. Manejo de la retinopatía diabética. 
El edema macular diabético traccional (EMDT) se caracteriza en la OCT por engrosamiento macular con pérdida de la depresión foveal y edema de las capas retinianas externas. La hialoides posterior en la OCT es gruesa e hiperreflactiva, está tensa y parcialmente desprendida del polo posterior, pero permanece aplicada a la papila y a la cima de la superficie macular elevada. La hialoides posterior engrosada y tensa ejerce una tracción tangencial vitreomacular que induce o exacerba el EMD.

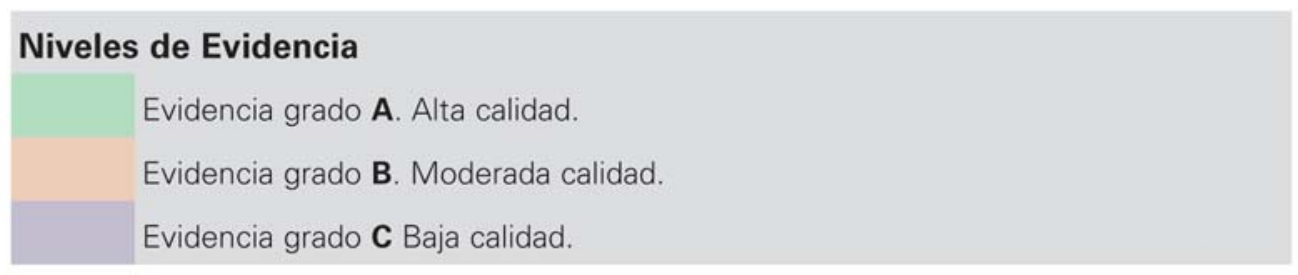

\section{Grados de Recomendación}

1 Fuerte recomendación ya que los beneficios superan claramente los riesgos.

2

Sugerencias de tratamientos en la que los riesgos y beneficios están más próximos o son más inciertos.

\section{Casos Especiales}

Con adherencias vitreoretinianas extensas: CRV con PFC se puede usar antiVEGF previos + CRV antes del 3 . $^{\text {er }}$ día \pm antiVEGF.

Papilopatía diabética traccional: CRV.

Maculopatía traccional: CRV.

Rubeosis iridiana: PFC extensa \pm antiVEGF.

AntiVEGF $\left({ }^{*}\right)$ en caso de: Hemorragia persistente o muy severa.

Se inyectaran cada 4 semanas manteniendo una estrecha vigilancia por riesgo de DRT. Si no mejora: CRV tras antiVEGF: no más del 3 . $^{\text {er }}$ día.

\section{RDP CON HEMORRAGIA PREMACULAR-RETROHIALOIDEA}

CRV antes de un mes.

\section{RDP CON HEMOVÍTREO RECURRENTE TRAS CRV}

Sangrado Inmediato: controles ecográficos. Si no se reabsorbe espontáneamente:

Cirugía Retinovitrea en 2-4 meses.

AntiVEGF preoperatorio.

Opción antiangiogénica: antiVEGF: cada 4-6 semanas. Control ecográfico cada 15 días.

Si mejora: completar PFC/Crioterapia periferia extrema.

Sangrado Tardío:

CRV + Crioterapia en esclerotomías + antiVEGF + gas electivo.

Opción antiangiogénica.

\section{RDP CON DESPRENDIMIENTO DE RETINA}

Traccional que afecte o amenace mácula: CRV + tamponador electivo (gas/aceite de silicona).

Regmatógeno/mixto: CRV $<48$ horas + aceite de silicona.

Gráfico 1 (continuación). Manejo de la retinopatía diabética. 
La biomicroscopía, a diferencia del OCT es insuficientemente precisa para determinar el estado de la hialoides posterior cuando ésta está sólo ligeramente desprendida de la superficie macular. La OCT es por ello más sensible que la biomicroscopía para identificar la adhesión vitreomacular y permite un diagnóstico más precoz de un desprendimiento parcial de vítreo posterior. Además permite valorar de forma precisa el engrosamiento macular teniendo una gran reproducibilidad.

\subsection{Opciones Terapéuticas}

\subsubsection{EMCS focal o multifocal}

El tratamiento de elección de este tipo de edema continúa siendo la fotocoagulación focal. Ofrece buenos resultados a largo plazo y está considerado como el tratamiento de referencia (figs. 4 y 5).

El tratamiento se realizará sobre los microaneurismas que fuguen y se encuentren situados en el centro de las coronas circinadas, entre 500 y 3.000 micras del centro de la ZAF, con spot de 50-100 micras y potencia suficiente para conseguir un blanqueo suave de los mismos.

En aquellos edemas, generalmente ya de tipo mixto (y no puramente focales), en los que el engro-

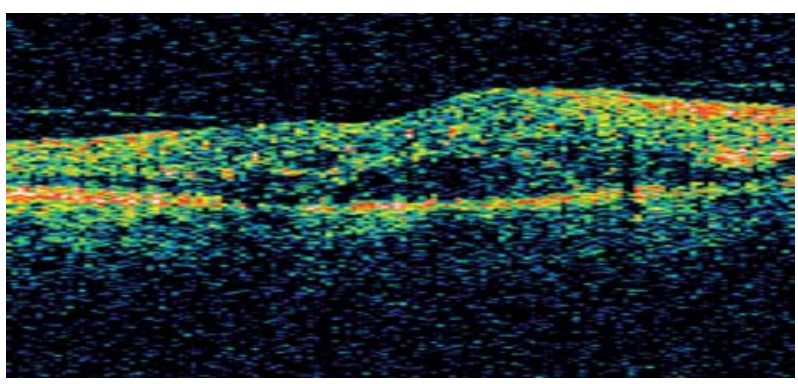

Fig. 14: Edema macular con síndrome de tracción vítreomacular.

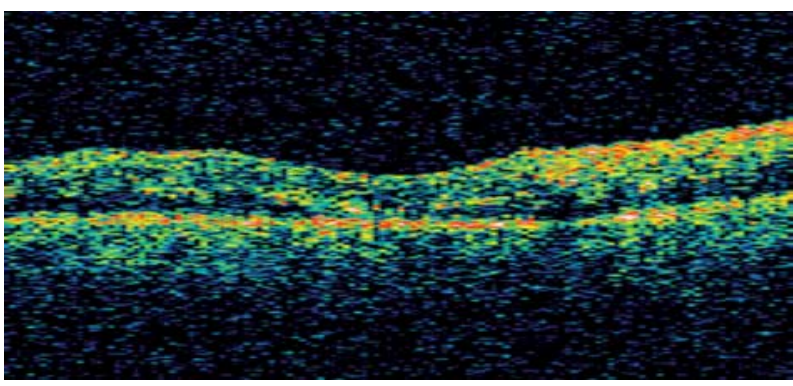

Fig. 15: El mismo caso tras cirugía retinovítrea. samiento macular central dificulte la laserterapia (que suele corresponder a valores de OCT superiores a $400 \mu$ (44) podría considerarse terapia antiangiogénica o corticoterapia intravítrea seguida de láser (45-49). Este tema se discute más ampliamente en el EMCS difuso.

En caso de que se objetive tracción vítreo-macular con repercusión funcional se procedería a CRV (5052) (figs. 7a y 7b) con o sin disección de la membrana limitante interna (MLI) (53-55) y/o TAIV/antiVEGF (44-48). Si se produce una mejoría estable el paciente pasa a control por su oftalmólogo.

\subsubsection{EMCS Difuso}

Cuando existe un EMCS difuso con tracción vítreorretiniana la indicación terapéutica es la CRV con hialodectomía (56-57) siendo discutida la disección de la MLI (58-63) así como el uso al final de la cirugía de TAIV $(64,65)$ (figs. 6, 14 y 15).

Si no existe tracción, la opción actualmente en vigor y que forma parte de los protocolos establecidos, es la aplicación de láser en rejilla modificada. En esta última se usan quemaduras menos intensas (gris suave) y más pequeñas $(50 \mu \mathrm{m})$ y se trata los microaneurismas de forma directa pero sin intentar conseguir el cambio de coloración de los mismos. Además trata zonas de engrosamiento retiniano y áreas de no perfusión que se crean en relación con el edema. El mapa de OCT es útil para identificar las zonas de engrosamiento retiniano. De acuerdo con los criterios del ETDRS el tratamiento debe repetirse a los 3-4 meses si el edema no se ha resuelto (máximo 3 tratamientos).

Los resultados que se obtienen con esta técnica son inferiores a los del edema macular focal. Sólo se evita la pérdida moderada (pérdida igual o mayor a 15 letras del ETRDS) de agudeza visual en un

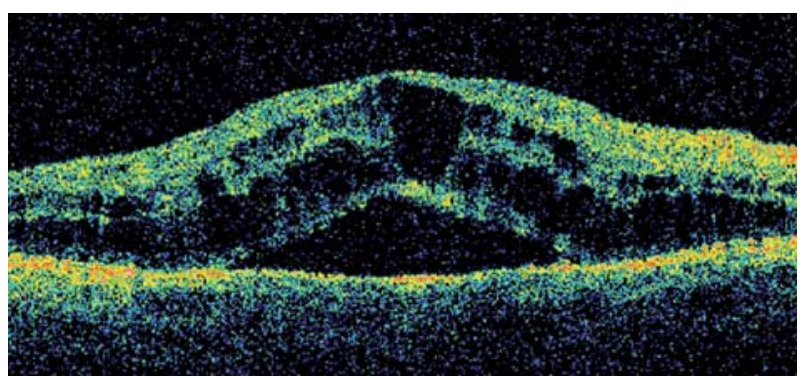

Fig. 16: OCT E3 de un paciente con EMCS difuso y gran engrosamiento macular central. 


\section{EDEMA MACULAR FOCAL Y MULTIFOCAL}

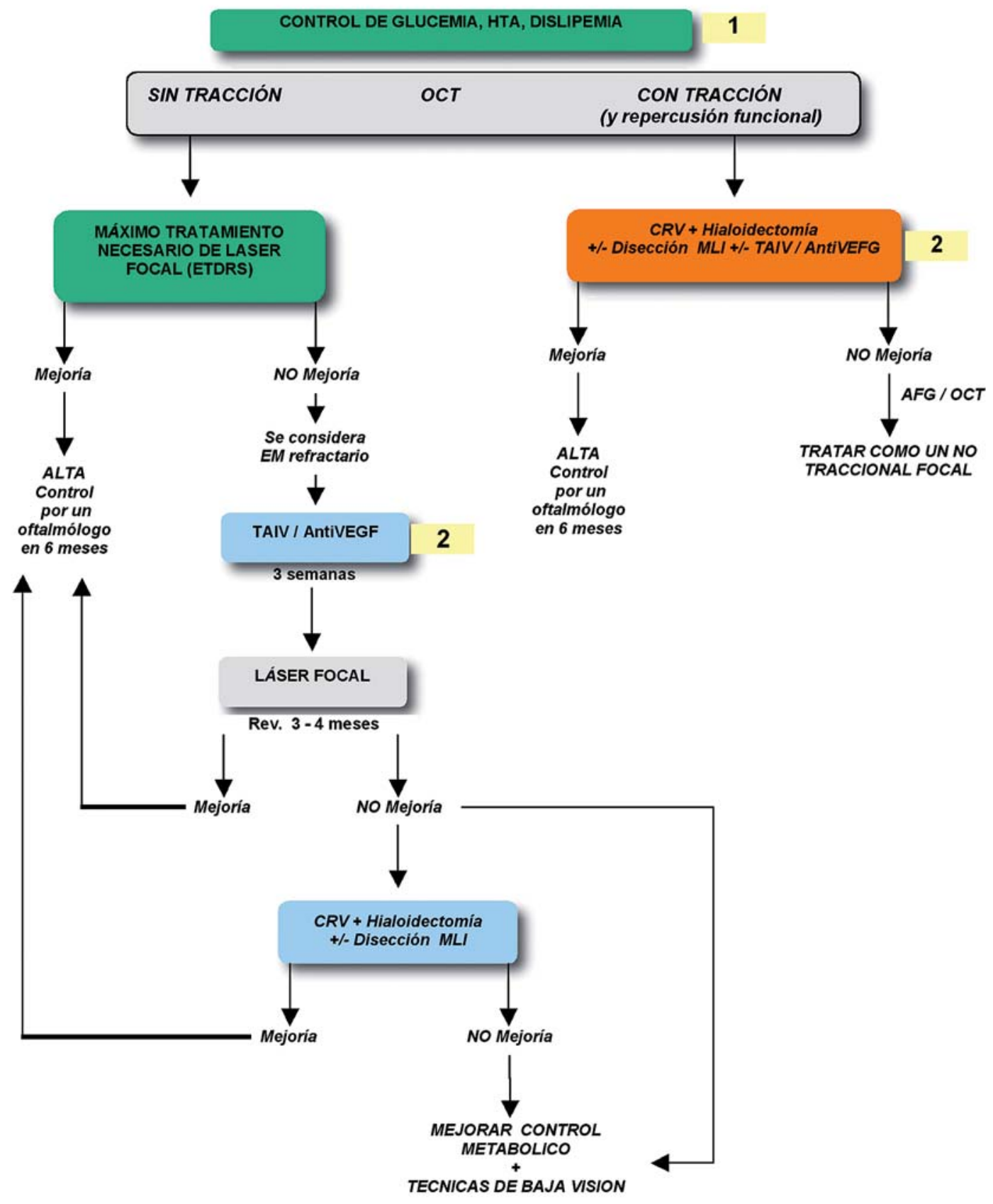

Gráfico 2. Algoritmo de tratamiento del edema macular focal y multifocal.

$50 \%$ de los pacientes, un $26 \%$ continua perdiendo visión a largo plazo y sólo un $3 \%$ experimenta una leve mejoría en su agudeza visual, por lo que se están planteando nuevas alternativas terapéuticas de las que sólo se dispone de datos preliminares ya que se encuentran en fase de investigación clínica.

Entre las alternativas farmacológicas propuestas para el control del edema macular difuso están la 


\section{EDEMA MACULAR DIFUSO / MIXTO}

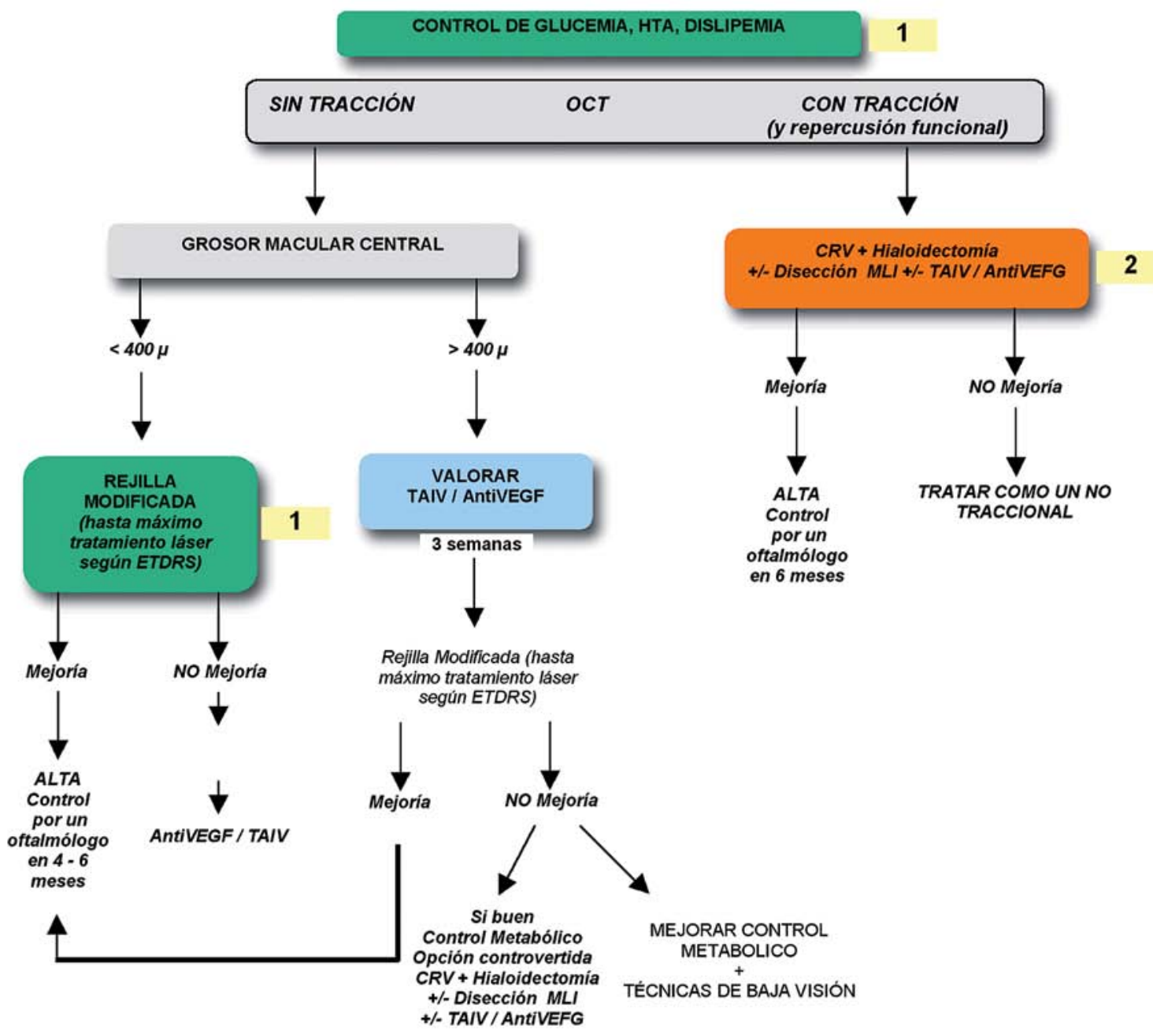

Gráfico 3. Algoritmo de tratamiento del edema macular difuso.

triamcinolona intravítrea y los nuevos fármacos antiVEGF. Con estas terapias se persigue una disminución de la difusión concretándose en algunas zonas en las que realizar láser terapia de forma más selectiva.

Ambas opciones exigen la solicitud de uso compasivo al Ministerio de Sanidad tanto si se emplean de forma más aislada.

Las complicaciones descritas con el uso de la TAIV (65-68) (glaucoma, DR, cataratas, endoftalmitis...) y los problemas legales que pueden derivar- se de su uso hacen que cada vez seamos más restrictivos en cuanto a la selección del paciente. En EMCS difusos con gran engrosamiento macular central (fig. 16), su eficacia lo convierte en el fármaco más recomendable. La opción más extendida es el uso de $4 \mathrm{mg}$ de TAIV en pacientes no vitrectomizados y $8 \mathrm{mg}$ en los vitrectomizados (69). En casos en que este engrosamiento macular central no sea tan marcado, nos inclinaríamos por los antiVEGF (bevacizumab, ranimizumab, pegaptanib...) cuyos efectos secundarios no parecen ser tan impor- 


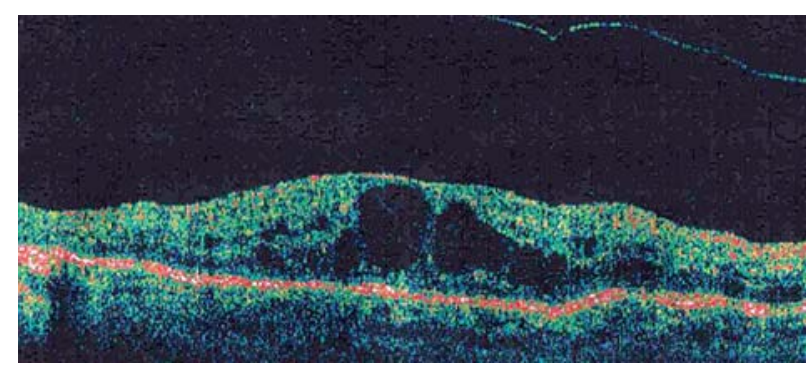

Fig. 17: Edema macular quístico no traccional.

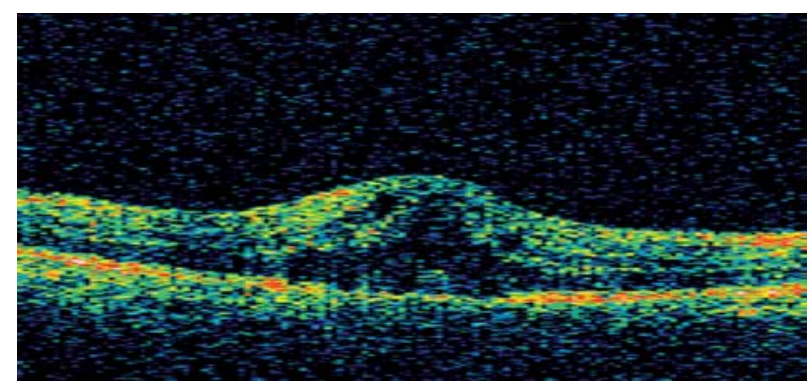

Fig. 18: Edema macular quístico.

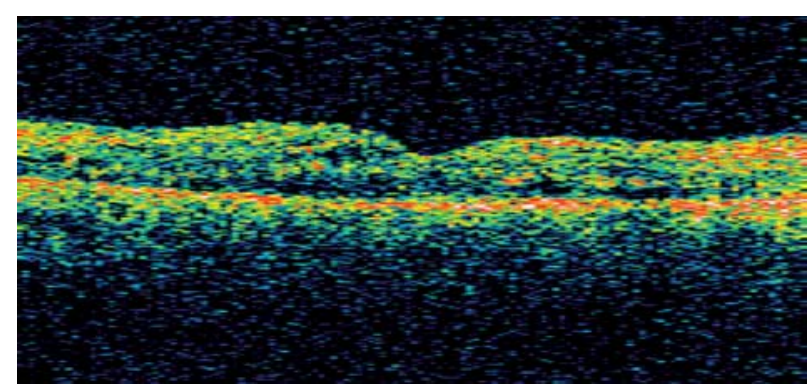

Fig. 19: El mismo EMQ tras TAIV.

tantes. Dado que se ha visto que el efecto de la TAIV es transitorio (70-73) se ha planteado la posibilidad de iniciar el tratamiento con una inyección de TAIV y continuarlo con un antiVEGF y/o con laserterapia (74-76).

Si tras las terapias propuestas no se obtuviera una mejoría, las opciones terapéuticas son reducidas y sólo en casos muy seleccionados se realizará CRV $(77,78)$ ya que hay estudios que no encuentran mejoría $(79,80)$.

\subsubsection{Edema macular quístico}

Ante un Edema Macular Quístico (EMQ) con tracción vitreomacular se propone CRV sin disec- ción de la MLI en aquellos casos en que se desconozca el tiempo de evolución o que éste sea superior a 6 meses (por el riesgo de inducir un agujero macular). En casos de que sea inferior a 6 meses podría realizarse la disección de la MLI.

En los pacientes afectos de EMQ en los que no exista evidencia de tracción, la opción que se nos plantea es el uso de TAIV seguido, de forma opcional, de antiVEGF y/o rejilla modificada $(81,83)$ (figs. 17-19).

A modo de resumen hacer hincapié que en presencia de un componente traccional, con repercusión funcional, en cualquier tipo de edema macular diabético, valoraremos la indicación de CRV.

\subsubsection{Edema Macular Isquémico}

La isquemia macular viene definida por signos clínicos como:

- Incremento de la zona avascular foveal (ZAF) $\geq 1.000 \mu \mathrm{m}$.

- Rotura del anillo capilar perifoveolar en el borde de la ZAF.

- Área de no perfusión dentro de un diámetro de disco del centro de la fóvea.

En el EMD de predominio isquémico con tracción se plantea la posibilidad de una CRV. El uso de antiVEGF está inicialmente contraindicado, pues aunque se logra un descenso del edema, la agudeza visual empeora por el incremento de la isquemia (84).

\subsubsection{Depósito lipídico masivo}

En algunos pacientes con edema macular crónico los depósitos lipídicos se concentran en área macular dañando de forma irreversible los fotorreceptores haciendo, por ello, ineficaz cualquier tratamiento.

\section{BIBLIOGRAFÍA}

1. Bloomgarden TZ. Diabetic retinopathy. Diabetes Care 2008; 31: 1080-1083.

2. Grading diabetic retinopathy from stereoscopic color fundus photographs — an extension of the modified Airlie House classification. ETDRS report number 10. Early treatment diabetic retinopathy study research group. Ophthalmology 1991; 98: 786-806.

3. Wilkinson CP, Ferris FL 3rd, Klein RE, Lee PP, Agardh $C D$, Davis $M$, et al. Proposed international clinical diabe- 
tic retinopathy and diabetic macular edema disease severity scales. Ophthalmology 2003; 110: 1677-1682.

4. Focal photocoagulation treatment of diabetic macular edema. Relationship of treatment effects to fluorescein angiographic and other retinal characteristics at baseline: ETDRS report no. 19. Early treatment diabetic retinopathy study research group. Arch Ophthalmol 1995; 113: 11441155.

5. Sander B, Larsen M, Engler C, Strom C, Moldow B, Larsen $N$, et al. Diabetic macular oedema: a comparison of vitreous fluorometry, angiography, and retinopathy. $\mathrm{Br} J$ Ophthalmol 2002; 86: 316-320.

6. Zein WM, Noureddin BN, Jurdi FA, Schakal A, Bashshur ZF. Panretinal photocoagulation and intravitreal triamcinolone acetonide for the management proliferative diabetic retinopathy with macular edema. Retina 2006; 26: 137-142.

7. Kumar A, Sinha S, Azad R, Sharma YR, Vohra R. Comparative evaluation of vitrctomy and dye-enhanced ILM peel with grid laser in diffuse diabetic macular edema. Graefes Arch Clin Exp Ophthalmol 2007; 245: 360-368.

8. Lee CM, Olk RJ. Modifies grid laser photocoagulation for diffusse diabetic macular edema. Long term visual results. Ophthalmology 1991; 98: 1594-1602.

9. Lee CM, Olk RJ, Akduman L. Combined modified grid and panretinal photocoagulation for diffusse diabetic macular edema and proliferative diabetic retinopathy. Ophthalmic Surg Lasers 2000; 31: 292-300.

10. Akduman L, Olk RJ. Subthreshold (invisible) modified grid and diodo laser photocoagulation in diffusse diabetic macular edema (DDME). Ophthalmic Surg Lasers 1999; 30: 706-714.

11. Akduman L, Olk RJ. Laser photocoagulation of diabetic macular edema. Ophthalmic Surg Lasers 1997; 28: $387-$ 408.

12. Panozzo G, Parolini B, Gusson E, Mercanti A, Pinackatt S, Bertoldo G, et al. Diabetic macular edema: an OCT-based classification. Semin Ophthalmol 2004; 19: 13-20.

13. American Diabetes Association. Diabetes Care. 2008; 31 : S1-S2.

14. Olafsdóttir E, Stefánsson E. Biennial eye screening in patients with diabetes without retinopathy: 10-year experience. Br J Ophthalmol 2007; 91: 1599-1601.

15. Progression of retinopathy with intensive versus conventional treatment in the Diabetes Control and Complications Trial. Diabetes Control and Complications Trial Research Group. Ophthalmology 1995; 102: 647-661.

16. Retinopathy and nephropathy in patients with type 1 diabetes four year after a trial of intensive therapy. Diabetes Control and Complications Trial/Epidemiology of Diabetes Interventions and Complications Research Group. $N$ England J Med 2003; 42: 381-389.

17. The relationship of glycemic exposure $(\mathrm{HbAlc})$ to the risk of development and progression of retinopathy in the diabetes control and complications trial. Diabetes 1995; 44: 968-983.

18. The effect of intensive treatment of diabetes on development and progression of long-term complications in insulin-dependent diabetes mellitus. Diabetes Control and Complications Trial Research Group. N Engl J Med 1993; 329: 977-986.

19. Intensive blood-glucose control with sulphonylureas or insulin compared with conventional treatment and risk of complications in patients with type 2 diabetes (UKPDS33). UK Prospective Diabetes Study (UKPDS) Group. Lancet 1998; 352: 837-853.

20. Tight blood pressure control and risk of macrovascular and microvascular complications in type 2 diabetes: UKPDS 38. UK Prospective Diabetes Study (UKPDS) Group. BMJ 1998; 317: 703-713.

21. Verdaguer Tarradella J. Clasificación de la Retinopatía diabética. En: Alfaro V, Gómez-Ulla F, Quiroz-Mercado H, Figueroa MS. Villalba SJ. Retinopatía diabética. Tratado médico quirúrgico. Madrid: Mac Line, S.L. 2006; 43-50.

22. Shimura $M$, Yasuda K, Nakazawa T, Kano T, Ohta S, Tamai M. Quantifying alterations of macular thickness before and after panretinal photocoagulation in patients with severe diabetic retinopathy and good vision. Ophthalmology 2003; 110: 2386-2394.

23. Sothornwit N. Intravitreal bevacizumab for ahmed glaucoma valve implantation in neovascular glaucoma: a case report. J Med Assoc Thai 2008; 91: S162-S165.

24. Mason JO 3rd, Yunker JJ, Vail R, McGwin G Jr. Intravitreal bevacizumab (Avastin) prevention of panretinal photocoagulation-induced complications in patients with severe proliferative diabetic retinopathy. Retina 2008; 28: 1319-1324.

25. National Eye Institute (NEI). Laser-Ranibizumab-Triamcinolone for Proliferative Diabetic Retinopathy (LRTforDME+PRP). Clinical Trials gov Identifier: NCT00445003, July 2008

26. Álvarez López A, Canut Jorda, MI. Estudio de bevacizumab (Avastin) intravítreo en el tratamiento del glaucoma neovascular. Anales Instituto Barraquer 2008, 37: 75-85.

27. Ruiz-Moreno JM, Montero JA, Lugo F, Amat P, Staicu C. Intravitreal bevacizumab in recurrent diabetic vitreous haemorrhage after vitrectomy. Acta Ophthalmol 2008; 86: 231-232.

28. Jonas JB, Libondi $T$, von Baltz $S$, Vossmerbaeumer $U$. Intravitreal bevacizumab for vitreous haemorrhage. Acta Ophthalmol 2008; 86: 585-586.

29. Spaide RF, Fisher YL. Intravitreal bevacizumab (Avastin) treatment of proliferative diabetic retinopathy complicated by vitreous hemorrhage. Retina 2006; 26: 275-278.

30. Arévalo F, Alfaro V, García RA, Fernández CF. Complicaciones de la vitrectomía en el paciente diabético. En: Alfaro V, Gómez-Ulla F, Quiroz-Mercado H, Figueroa MS. Villalba SJ. Retinopatía diabética. Tratado médico quirúrgico. Madrid: MacLine, S.L. 2006; 445-462.

31. Ruiz-Moreno JM, Montero JA, Lugo F, Amat P, Staicu C. Intravitreal bevacizumab in recurrent diabetic vitreous haemorrhage after vitrectomy. Acta Ophthalmol 2008; 86: 231-232.

32. Berrocal MH. Proliferación Fibrovascular Hialoidea Anterior. En: Alfaro V, Gómez-Ulla F, Quiroz-Mercado H, Figueroa MS. Villalba SJ. Retinopatía diabética. Tratado médico quirúrgico. Madrid: MacLine, S.L. 2006; 425-429.

33. Engelbert M, Del Priore L, Al-Aswad A. Neovascular Glaucoma. Contemporary Ophthalmology 2008; 7: 15.

34. Hong C, Arosemena A, Zurakowowski D, Ayyala RS. Glaucoma drainage devices: a sistematic literature review and current controversies. Diagnostic and surgical techniques. Surv Ophthalmol 2005; 50: 48-60.

35. Ichhpujani P, Ramasubramanian A, Kaushik S, Pandav S. Bevacizumab in glaucoma: a review. Can Ophthalmol 2007; 42: 812-815. 
36. Mirshahi A, Roohipoor $R$, Lashay A, Mohammadi SF Abdoallahi A, Faghihi H. Bevacizumab-augmented retinal laser photocoagulation in proliferative diabetic retinopathy: a randomized double-masked clinical trial. Eur J Ophthalmol 2008; 18: 263-269.

37. Schlote T, Derse M, Rassmann K, Nicaeus T, Dietz K, Thiel HJ. Efficacy and safety of contact transscleral diode laser cyclophotocoagulation for advanced glaucoma. J Glaucoma 2001; 10: 294-301.

38. Miki A, Oshima Y, Otori Y, Kamei M, Tano Y. Efficacy of intravitreal bevacizumab as adjunctive treatment with pars plana vitrectomy, endolaser photocoagulation, and trabeculectomy for neovascular glaucoma. Br J Ophthalmol 2008; 92: 1431-1433.

39. Kitnarong N, Chindasub P, Metheetrairut A. Surgical outcome of intravitreal bevacizumab and filtration surgery in neovascular glaucoma. Adv Ther 2008; 25: 438-443.

40. European Glaucoma Society Terminology and guidelines for glaucoma. Italy: Dogma. 2008.

41. Jurecka T, Bátková Z, Ventruba J, Synek S. Macular edema after cataract surgery in diabetic patients without retinopathy. Cesk Slov Oftalmol 2007; 63: 274-284.

42. Macugen to prevent worsening of macular edema following cataract surgery in diabetics. Information provided by: Johns Hopkins University. Clinical Trials Gov Identifier: NCT00346983.

43. Triamcinolona. Clinical Trial Gov Identifier: NCT00801450.

44. Estabrook EJ, Madhusudhana KC, Hannan SR, Newsom $R S$. Can optical coherence tomography predict the outcome of laser photocoagulation for diabetic macular edema? Ophthalmic Surg Lasers Imaging 2007; 38: 478-483.

45. Kaderli B, Avci R, Gelisken O, Yucel AA. Intravitreal triamcinolone as an adjunct in the treatment of concomitant proliferative diabetic retinopathy and diffuse diabetic macular edema: combined IVTA and laser treatment for PDR with CSMO. Int Ophthalmol 2005; 26: 207-214.

46. Lida T. Combined triamcinolone acetonide injection and grid laser photocoagulation: a promising treatment for diffuse diabetic macular edema? Br J Ophthalmol 2007; 91: 407-408.

47. Shimura M, Nakazawa T, Yasuda K, Shiono T, Nishida K. Pretreatment of posterior subtenon of triamcinolone acetonide has beneficial effects for gird pattern photocoagulation against diffuse diabetic macular oedema. $\mathrm{Br} \mathrm{J} \mathrm{Oph}$ thalmol 2007; 91: 449-454.

48. Kang SW, Sa HS, Cho HY, Kim JL. Macular gird photocoagulation after intravitreal triamcinolone acetonide for diffuse diabetic macular edema. Arch Ophthalmol 2006; 124: 653-658.

49. Haritoglou C, Kook D, Neubauer A, Wolf A, Priglinger S, Strauss R. Intravitreal bevacizumab (Avastin) therapy for persistent diffuse diabetic macular edema. Retina 2006; 26: 999-1005.

50. Cabrera López F, Cabrera Marrero B, Baeta Bayón L, Jerez Olivera E, Cardona Guerra P. Actualización en el diagnóstico y tratamiento del edema macular diabético. Arch Soc Can Oftalmol 2005; 16: 69-82.

51. Lewis H, Abrams GW, Blumenkranz MS, Campo RV. Vitrectomy for diabetic macular traction and edema associated with posterior hyaloidal traction. Ophthalmology 1992; 99: 753-759.
52. Harbour JW, Smiddy WE, Flynn HW Jr, Rubsamen PE. Vitrectomy for diabetic macular edema associated with thickened and taut posterior hyaloid membrane. Am J Ophthalmol 1996; 121: 405-413.

53. Gandorfer A, Messmer EM, Ulbig MW, Kampik A. Resolution of diabetic macular edema after surgical removal of than posterior hyaliod and the inner limiting membrane. Retina 2000; 20: 126-133.

54. Avic R, Karderli B, Avic B, Simsek S, Baykara M, Kahveci $Z$, et al. Pars plana vitrectomy and removal of the internal limiting membrane in the treatment of chronic macular edema. Graefes Arch Clin Exp Ophthalmol 2004; 242: 845-852.

55. Recchia FM, Ruby AJ, Recchia CA. Pars plana vitrectomy with removal of the internal limiting membrane in the treatment of persistent diabetic macular edema. Am J Ophthalmol 2005; 139: 447-454.

56. Pendergast SD, Hassan TS, Williams GA, Cox MS, Margherio RR, Ferrone PJ, et al. Vitrectomy for diffuse diabetic macular edema associated with taut premacular posterior hyaloid. Am J Ophthalmol 2000; 130: 178-186.

57. Lövestam-Adran M, Larsson J. Vitrectomy seems to be beneficial for advanced diffuse diabetic macular oedema not responding to laser treatment. Int Ophthalmol 2005; 26: 21-26.

58. Matsumoto H, Yamanaka I, Hisatomi T, Enaida H, Ueno A, Hata $Y$, et al. Triamcinolone assisted pars plana vitrectomy improves residual posterior vitreus hyaloid removal: ultrastructural analysis of the inner limiting membrane. Retina 2007; 27:174-179.

59. Bahadir M, Ertan A, Mertoglu O. Visual acuity comparision of vitrectomy with and without internal limitig membrane removal in the treatment of diabetic macular edema. Int Ophthalmol 2005; 26: 3-8.

60. Patel JI, Hykin PG, Schadt M, Luong V, Fitzke F, Gregor ZJ. Pars plana vitrctomy with and without peeling of the inner limiting membrane for diabetic macular edema. Retina 2006; 26: 5-13.

61. Aboutable T. Is removal of internal limiting membrane always necessary during surgery for refractory diffuse diabetic macular edema without evident epimacular proliferation? Klin Mmonatsbl Augenheilkd 2006; 223: 681-686.

62. Meyer $\mathrm{CH}$. Current treatment approaches in diabetic macular edema. Opthalmologica 2007; 221: 118-131.

63. Browning DJ, Fraser CM, Powers ME. Comparison of the magnitude and time course of macular thinning induced by different interventions for diabetic macular edema: implications for sequence of application. Ophthalmology 2006; 113: 1713-1719.

64. Yanyali A, Nohutcu AF, Horozoglu F, Celik E. Modified grid laser photocoagulation versus pars plana vitrectomy with internal limiting membrane removal in diabetic macular edema. Am J Ophthalmol 2005; 139: 795-801.

65. Jonas JB. Intravitreal triamcinolone acetonide: a change in paradigm. Ophthalmic Res 2006; 38: 218-245.

66. Jonas JB, Degenring RF, Kreissig I, Akkoyun I, Kampeter $B A$. Intraocular pressure elevation after triamcinolone acetonide injection. Ophthalmology 2006; 112: 593-598.

67. Kreissig I, Degenring RF, Jonas JB. Diffuse diabetic macular edema. Intraocular pressure after intravitreal triamcinolone acetonide. Ophthalmologe 2005; 102: 153157. 
68. Jonas, Kreissig I, Budde WM, Degenring RF. Cataract surgery combined with intravitreal triamcinolone acetonide. Eur J Ophthalmol 2005; 15: 329-335.

69. Chin HS, Park TS, Moon YS, Oh JH. Difference in clearance of intravitreal triamcinolone acetonide between vitrectomized and nonvitrectomized eyes. Retina 2005; 25: 556-560.

70. Jonas JB. Intravitreal triamcinolone acetonide for treatment of intraocular oedematous and neovascular diseases. Acta Ophthalmol Scand 2005; 83: 645-663.

71. Spandau UH, Derse M, Schmiz-Valckemberg P, Papoulis $C$, Jonas JB. Dosage dependency of intravitreal triamcinolone acetonide as treatment of diabetic macular oedema. Br J Ophthalmol 2005; 89: 999-1003.

72. Gibran SK, Cullinae A, Jungkim S, Cleary PE. Intravitreal triamcinolone for diffuse diabetic macular edema. Eye 2000; 20: 720-724.

73. Jonas JB, Degenring RF, Kamppeter BA, Kreissig I, Akkoyun J. Duration of the effect of intravitreal triamcinolone acetonide as treatment for diffuse diabetic macular edema. Am J Ophthalmol 2004; 138: 158-160.

74. A Multicentre Randomised Clinical Trial of Laser Treatment Plus Intravitreal Triamcinolone for Diabetic Macular Oedema. Clinical Trial Gov Identifier: NCT00148265.

75. Efficacy and Safety Study of Intravitreal Triamcinolone to Treat Diffuse Diabetic Macular Edema. Clinical Trial Gov Identifier: NCT00309192.

76. Laser-Ranibizumab-Triamcinolone for Diabetic Macular Edema (LRT for DME). Clinical Trial Gov Identifier: NCT00444600.
77. Tachi N, Ogino N. Vitrectomy for diffuse diabetic macular edema in cases of diabetic retinopathy. Am J Ophthalmol 1996; 122: 258-260

78. Otani T, Kishi S. A cotrolled study of vitrectomy for diabetic macular edema. Am J Ophthalmol 2002; 134: 214 219.

79. Figueroa MS, Contreras I, Noval S. Surgical and anatomical outcomes of pars plana vitrectomy for diffuse nontractional diabetic macular edema. Retina 2008; 28: 420426.

80. Hartley KL, Smiddy WE, Flynn HW Jr, Murray TG. Pars plana vitrectomy with internal limiting membrane peeling for diabetic macular edema. Retina 2008; 28:410-419.

81. Avitabile T, Longo A, Reibaldi A. Intravitreal triamcinolone compared with macular laser grid photocoagulation for the treatment of cystoid macular edema. Am J Ophthalmol 2005; 140: 695-698.

82. Diabetic Retinopathy Clinical Research Network. A randomized trial comparing intravitreal triamcinolone acetonide and focal/grid photocoagulation for diabetic macular edema. Ophthalmology 2008; 115: 1447-1449.

83. Ho TC, Lai WW, Lam DS. Intravitreal triamcinolone compared with macular laser grid photocoagulation for the treatment of cystoid macular edema. Am J Ophthalmol 2006; 141: 786-787.

84. Chung EJ, Roh MI, Kwon OW, Koh HJ. Effects of macular ischemia on the outcome of intravitreal Bevacizumab therapy for diabetic macular edema. Retina 2008; 28: 957-963. 